\title{
Mode competition in a system of two parametrically driven pendulums; the role of symmetry
}

\author{
E.J. Banning ${ }^{1}$, J.P. van der Weele*, M.M. Kettenis ${ }^{2}$ \\ Center for Theoretical Physics, University of Twente,P.O. Box 217, 7500 AE Enschede, \\ The Netherlands
}

Received 5 June 1997

\begin{abstract}
This paper is the final part in a series of four on the dynamics of two coupled, parametrically driven pendulums. In the previous three parts (Banning and van der Weele, Mode competition in a system of two parametrically driven pendulums; the Hamiltonian case, Physica A 220 (1995) 485-533; Banning et al., Mode competition in a system of two parametrically driven pendulums; the dissipative case, Physica A 245 (1997) 11-48; Banning et al., Mode competition in a system of two parametrically driven pendulums with nonlinear coupling, Physica A 245 (1997) 49-98) we have given a detailed survey of the different oscillations in the system, with particular emphasis on mode interaction. In the present paper we use group theory to highlight the role of symmetry. It is shown how certain symmetries can obstruct period doubling and Hopf bifurcations; the associated routes to chaos cannot proceed until these symmetries have been broken. The symmetry approach also reveals the general mechanism of mode interaction and enables a useful comparison with other systems.
\end{abstract}

Keywords: Mode competition; Symmetry; Pendulums

\section{Introduction}

\subsection{Outline}

This is the fourth and final part of a series on the dynamics of two coupled, parametrically driven pendulums. In the three previous papers [1-3] we made a detailed

\footnotetext{
* Corresponding author. E-mail: j.p.vanderweele@tn.utwente.nl.

' Present address: EPT-RF, Shell International Exploration and Production B.V., Volmerlaan 8, P.O. Box 60, 2280 AB Rijswijk, The Netherlands.

2 Present address: Institute for Theoretical Physics, University of Amsterdam, Valckenierstraat 65, $1018 \mathrm{XE}$ Amsterdam, The Netherlands.
} 
survey of the different oscillations associated (directly or indirectly) with the main parametric resonance of the system, paying particular attention to the phenomenon of mode competition. We have seen time and again that the dynamics, and especially the bifurcational behavior, can be understood to a large extent by considering the symmetries of the motions; in the present paper we shall use group theory to work out this notion. This will put the work on a somewhat higher level of abstraction, exposing the general rather than the system-specific features, thus enabling us to compare our results with those of others.

The most important oscillations in the two-pendulum system have period 2 with respect to the driving, as a consequence of the fact that the driving is parametric. They are related to each other by symmetry breaking bifurcations (which do not affect the periodicity) and can be arranged in a so-called isotropy lattice. It turns out that the dynamics of the system is largely covered by the motions in this period-2 isotropy lattice, since bifurcations that generate motions of higher period (period doubling bifurcations) and quasiperiodic motions (Hopf bifurcations) do not occur until relatively low in the lattice, when certain symmetries are no longer present.

When two motions have a common daughter in the symmetry hierarchy, mode interaction via this daughter becomes a distinct possibility. This phenomenon has been observed in many experiments [4-14]; the underlying principle is always the same, but the actual appearance of the interaction may vary widely from system to system. Mode interaction typically occurs when two mother motions are born simultaneously from another motion; the interaction region in parameter space then originates from the intersection point of the mother-motions' birthlines. In our case the intersecting birthlines are associated with period doubling bifurcations of a period- 1 motion (the trivial equilibrium motion). The two interacting modes, i.e., the mother motions, and their common daughter all have period 2 and hence the mode interaction takes place entirely within the period-2 isotropy lattice. In other systems the mother's birthlines may be associated with other kinds of bifurcation and the periodicity of the modes involved in the interaction may be different. But even for a system in which the birthlines are associated with period doublings as in our two-pendulum model, and even if it has exactly the same symmetry structure, small differences in the nonlinear terms of the equations of motion may cause the interaction to look quite dissimilar. A good example of this is provided by the compound pendulum of Skeldon and Mullin [6] and Skeldon [7], or simply by our own model with a different choice of (nonlinear) coupling [3].

The paper is built up as follows. In the remainder of this introduction we present the system and its symmetries. In Section Section 2 we discuss the Hamiltonian period-2 isotropy lattice. In the main text we restrict ourselves to the part corresponding to the motions that have actually been observed in practice, and in the appendix a more complete version of the Hamiltonian lattice is presented. In Section 3 we give the dissipative period-2 lattice, which turns out to be much simpler than its Hamiltonian counterpart. In the dissipative case, moreover, there is no discrepancy between the observed and the complete versions of the lattice. Along with the lattices we also discuss the mode interaction in our system. In Section 4 we examine the possible 


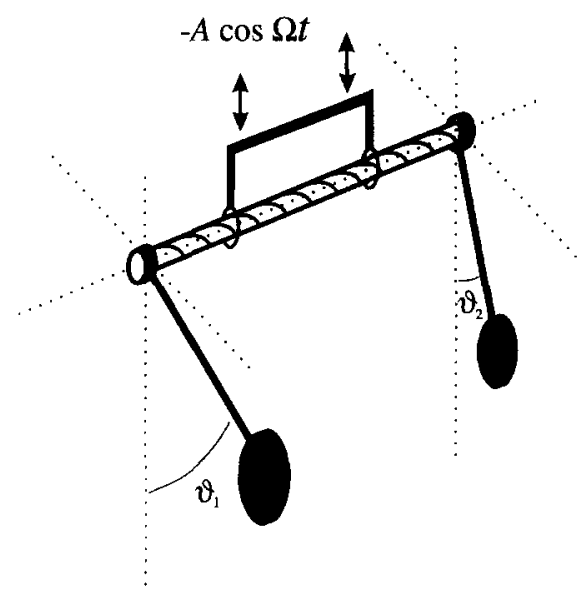

Fig. 1. The system, consisting of two parametrically driven (identical) pendulums, coupled by a torsion spring.

escape routes from the isotropy lattice, via period doubling and Hopf bifurcations. A comparison with other systems is given in Section 5 and finally, in Section 6, we summarize and make some concluding remarks.

\subsection{The system and its symmetries}

Our system consists of two pendulums, coupled by a torsion spring and subjected to a harmonic forcing in the vertical direction; this kind of forcing is known as parametric driving. The system is depicted in Fig. 1, and its equations of motion are given by

$$
\begin{aligned}
& \ddot{\vartheta}_{1}+f(t) \sin \vartheta_{1}+\gamma \dot{\vartheta}_{1}+F\left(\vartheta_{1}, \vartheta_{2}\right)=0, \\
& \ddot{\vartheta}_{2}+f(t) \sin \vartheta_{2}+\gamma \dot{\vartheta}_{2}-F\left(\vartheta_{1}, \vartheta_{2}\right)=0,
\end{aligned}
$$

where $f(t)$ contains the driving term:

$$
f(t)=\frac{1}{l}\left(g+A \Omega^{2} \cos \Omega t\right),
$$

the terms $\gamma \dot{\vartheta}_{1}$ and $\gamma \dot{\vartheta}_{2}$ represent viscous damping in the pivots (for $\gamma=0$ the system is Hamiltonian) and the function $F\left(\vartheta_{1}, \vartheta_{2}\right)$ stands for the coupling between the pendulums. The dissipation is deliberately chosen to be the standard viscous damping, linear in the angular velocities, in order to facilitate the comparison with other (theoretical) work on related systems; the coupling function, on the other hand, is allowed to be non-linear (cf. Eq. (1.5)). For more details on this model system please see Refs. $[1-3]$.

We are interested in the 'pure modes' (i.e., the oscillations bifurcating from the downward equilibrium motion along the borders of the first-order resonance tongues, as indicated in Fig. 2) and in the oscillatory motions that bifurcate from them. For a proper description of these oscillations we exploit the symmetries of the system. To 


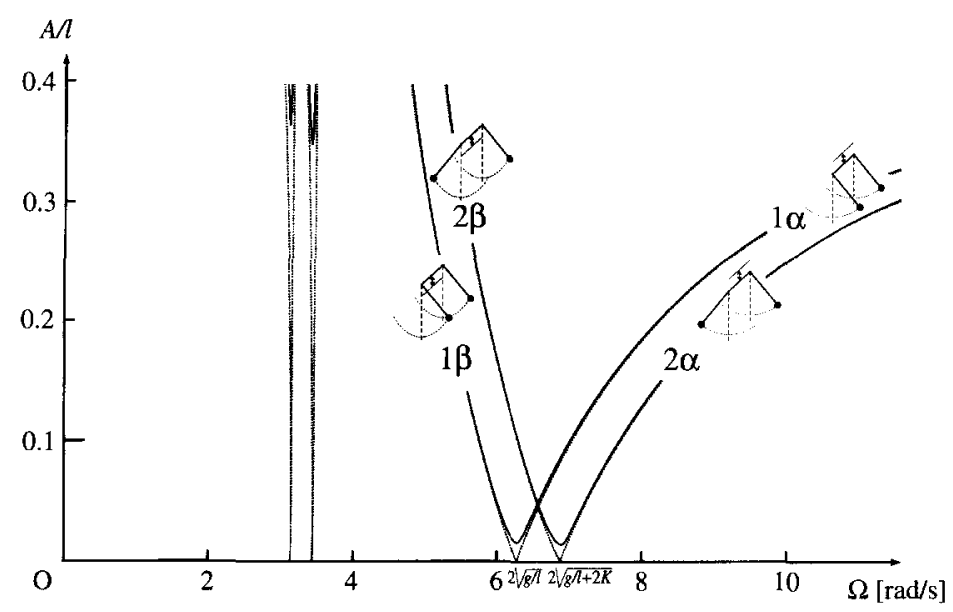

Fig. 2. The first- and second-order resonance tongues in the $(A, \Omega)$-plane, for any coupling with linear coefficient $K=1 \mathrm{~s}^{-2}$ (cf. Eq. (1.5)). The dotted and solid lines represent the Hamiltonian bifurcation lines $\left(\gamma=0 \mathrm{~s}^{-1}\right)$ and their dissipative counterparts $\left(\gamma=0.1 \mathrm{~s}^{-1}\right)$ respectively. The pure modes $1 \alpha, 2 \alpha, 1 \beta$ and $2 \beta$ are born along the borders of the first-order resonance tongues.

begin with, we need two of its spatial symmetries, namely reflection and pendulum exchange: ${ }^{3}$

$$
\begin{aligned}
& \boldsymbol{R}:\left(\vartheta_{1}, \dot{\vartheta}_{1}, \vartheta_{2}, \dot{\vartheta}_{2}, t\right) \rightarrow\left(-\vartheta_{1},-\dot{\vartheta}_{1},-\vartheta_{2},-\dot{\vartheta}_{2}, t\right), \\
& \boldsymbol{E}:\left(\vartheta_{1}, \dot{\vartheta}_{1}, \vartheta_{2}, \dot{\vartheta}_{2}, t\right) \rightarrow\left(\vartheta_{2}, \dot{\vartheta}_{2}, \vartheta_{1}, \dot{\vartheta}_{1}, t\right)
\end{aligned}
$$

(A symmetry is called 'spatial' if the corresponding operation does not affect the time coordinate.) It is easily checked that the equations of motion (Eq. (1.1)) are left unchanged by these operations if the coupling does not violate the spatial symmetries, that is, if the coupling satisfies $F\left(\vartheta_{1}, \vartheta_{2}\right)=-F\left(\vartheta_{2}, \vartheta_{1}\right)=-F\left(-\vartheta_{1},-\vartheta_{2}\right)$. We thus take (cf. Ref. [3]):

$$
F\left(\vartheta_{1}, \vartheta_{2}\right)=K\left(\vartheta_{1}-\vartheta_{2}\right)+L\left(\vartheta_{1}-\vartheta_{2}\right)^{3}+M\left(\vartheta_{1}-\vartheta_{2}\right)^{5}+\cdots
$$

Since both $\boldsymbol{R}$ and $\boldsymbol{E}$ are their own inverse we may write the associated part of the symmetry group of the system as $Z_{2}(R) \times Z_{2}(E)$; here $Z_{2}$ denotes the cyclic group of order 2 , consisting of the identity and one non-trivial element. Apart from these spatial symmetries, we also need the temporal symmetry associated with translation over one driving period:

$$
\boldsymbol{T}:\left(\vartheta_{1}, \dot{\vartheta}_{1}, \vartheta_{2}, \dot{\vartheta}_{2}, t\right) \rightarrow\left(\vartheta_{1}, \dot{\vartheta}_{1}, \vartheta_{2}, \dot{\vartheta}_{2}, t+T\right) \text {, with } T=2 \pi / \Omega \text {. }
$$

(A symmetry is called 'temporal' if it does not affect the angles $\theta_{1}$ and $\theta_{2}$.) If we restrict ourselves to motions that repeat themselves after every 2 periods of the driving (i.e., motions of period $T$ and $2 T$ ) we may identify $t+2 \cdot T$ with $t$, and then $T$ is also

\footnotetext{
${ }^{3}$ In particular, we do not need the rotational symmetry of the system to describe the motions we are interested in.
} 
a $Z_{2}$-symmetry. The symmetry group of the system, or at least the part relevant for our purposes, is then equal to

$$
Z_{2}(R) \times Z_{2}(E) \times Z_{2}(T)
$$

This group contains $2^{3}=8$ elements. In the special case of $\gamma=0$ (i.e., in the Hamiltonian case) the operation of time reversal

$$
\boldsymbol{t}:\left(\vartheta_{1}, \dot{\vartheta}_{1}, \vartheta_{2}, \dot{\vartheta}_{2}, t\right) \rightarrow\left(\vartheta_{1},-\dot{\vartheta}_{1}, \vartheta_{2},-\dot{\vartheta}_{2},-t\right)
$$

is also a symmetry of the system. Accordingly, for the Hamiltonian system (still restricting ourselves to $2 T$-periodic oscillations) the relevant symmetry group is

$$
Z_{2}(R) \times Z_{2}(E) \times Z_{2}(\boldsymbol{T}) \times Z_{2}(\boldsymbol{t}),
$$

which has $2^{4}=16$ group elements.

The symmetries of the system are not necessarily present in the pendulum oscillations. Indeed, $\boldsymbol{R}$ and $\boldsymbol{T}$ never appear as individual symmetries in the oscillations we are interested in (with the exception of the downward equilibrium motion). Generally, a motion of period 2, i.e., a solution to Eq. (1.1) that is periodic in time with period $2 T$, is said to possess a symmetry if all points of its orbit are invariant under the corresponding operation. For example, a motion $\bar{\theta}(t)$ possesses $\boldsymbol{R} \boldsymbol{T}$ symmetry if and only if the following relation holds for all $t$ :

$$
\bar{\theta}(t)=\boldsymbol{R} \boldsymbol{T} \bar{\theta}(t) \Leftrightarrow\left(\begin{array}{c}
\bar{\vartheta}_{1}(t) \\
\overline{\bar{\vartheta}}_{1}(t) \\
\bar{\vartheta}_{2}(t) \\
\overline{\bar{\vartheta}}_{2}(t)
\end{array}\right)=\boldsymbol{R} \boldsymbol{T}\left(\begin{array}{c}
\bar{\vartheta}_{1}(t) \\
\overline{\bar{\vartheta}}_{1}(t) \\
\bar{\vartheta}_{2}(t) \\
\dot{\bar{\vartheta}}_{2}(t)
\end{array}\right)=\left(\begin{array}{c}
-\bar{\vartheta}_{1}(t+T) \\
-\dot{\bar{\vartheta}}_{1}(t+T) \\
-\bar{\vartheta}_{2}(t+T) \\
-\dot{\bar{\vartheta}}_{2}(t+T)
\end{array}\right) .
$$

The symmetries of a motion form a subgroup (formally called an isotropy subgroup) of the system's full symmetry group. Now, when a motion undergoes a symmetry breaking bifurcation, the two newly born motions in general have a symmetry group that is a maximal subgroup of the original motion's group. (See also Ref. [15], in particular the discussion concerning the equivalent branching lemma.) In the $Z_{2}$-context of our system this means that, if the original motion has a symmetry group of the form

$$
Z_{2} \times Z_{2} \times Z_{2}
$$

its products under a symmetry breaking bifurcation will have a group (i.e., a maximal subgroup) of the form

$$
Z_{2} \times Z_{2}
$$

As stated before, we are interested in the downward equilibrium motion, the pure modes originating at the first-order resonance tongues (see Fig. 2) and their bifurcation products. Of all these motions, only the downward motion itself possesses all of the symmetries mentioned above; its symmetry group is therefore equal to Eq. (1.7), or in the Hamiltonian case (1.9). (In the context of this paper it is convenient to regard the 
downward motion as having periodicity 2 with respect to the driving, despite the fact that it actually repeats itself after every driving period.) The maximal subgroups of this group, together with their maximal subgroups, and so on, constitute the so-called isotropy lattice; this lattice represents all possible $2 T$-periodic oscillations born (directly or indirectly) from the downward equilibrium motion. In the next two sections we will construct this lattice both for the Hamiltonian and the dissipative case. Let us stress from the outset that the lattice we are about to present is not the complete isotropy lattice of the system (which is considerably larger, covering every motion possible in our two-pendulum system), nor even the complete lattice for oscillations originating from the downward equilibrium (which would also include the motions born at the higher-order resonance tongues), but only the sub-lattice corresponding to the $2 T$-periodic oscillations we are interested in.

\section{The Hamiltonian isotropy lattice}

The (sub)groups in the isotropy lattice, tabulated in Table 1, are divided over five levels. Each of the levels is characterized by a general group structure, as follows:

$$
\begin{array}{ll}
\text { level I : } Z_{2} \times Z_{2} \times Z_{2} \times Z_{2}, & \text { with } 16 \text { elements per group, } \\
\text { level II : } Z_{2} \times Z_{2} \times Z_{2}, & \text { with } 8 \text { elements per group, } \\
\text { level III : } Z_{2} \times Z_{2}, & \text { with } 4 \text { elements per group, } \\
\text { level IV : } Z_{2}, & \text { with } 2 \text { elements per group, } \\
\text { level V : } \mathbf{1 .} & \text { with } 1 \text { element (the identity). }
\end{array}
$$

The number of groups in each level may need clarification. In level I we find only one group, which corresponds to the trivial motion. The next level contains four groups, corresponding to the four 'pure modes' bifurcating from the trivial solution along the borders of the first-order resonance tongues. Each of these level II groups has seven daughters (maximal subgroups) in level III. However, this does not mean that the total number of groups in level III amounts to $4 \times 7=28$, since some of the daughters coincide. To be specific, every level II group shares one daughter with each of the three other level II groups. The total number of groups in level III thus equals $28-6=22$. The level III groups in turn each have 3 daughters in level IV; not all of them are distinct, and close inspection (we will come to this in the appendix) reveals that there are in fact only 13 different groups in level IV. Finally, the fifth level consists of one single group, namely the identity transformation 1; this is the common daughter of all 13 level IV groups. Adding up, we thus find a total $1+4+22+13+1=41$ groups, spread over five levels.

The 41 groups can be represented in an elegant, albeit complicated, three-dimensional graph; this is done in the appendix. In the present section we restrict ourselves to the groups that correspond to oscillations that have actually been observed. These are the 
Table 1

The groups of the Hamiltonian isotropy lattice

$$
\begin{aligned}
& \text { Level I } \\
& \text { Level II } \\
& Z_{2}(R T) \times Z_{2}(E) \times Z_{2}(t) \\
& Z_{2}(R T) \times Z_{2}(E T) \times Z_{2}(t) \\
& \text { Level III } \\
& Z_{2}(R T) \times Z_{2}(t) \\
& Z_{2}(R T) \times Z_{2}(T t) \\
& Z_{2}(R T) \times Z_{2}(E \mathbf{t}) \\
& Z_{2}(R T) \times Z_{2}(E T t) \\
& Z_{2}(R T) \times Z_{2}(E) \\
& Z_{2}(R T) \times Z_{2}(E T) \\
& Z_{2}(E) \times Z_{2}(t) \\
& Z_{2}(E) \times Z_{2}(T t) \\
& Z_{2}(E T) \times Z_{2}(t) \\
& Z_{2}(E T) \times Z_{2}(T t) \\
& Z_{2} \text { (RETt) } \times Z_{2} \text { (RTt) } \\
& Z_{2} \text { (RTt) } \times Z_{2} \text { (RET) } \\
& Z_{2}(\text { RET }) \times Z_{2} \text { (RETt) } \\
& Z_{2}(\text { REt }) \times Z_{2}(\text { Rt }) \\
& Z_{2}(R t) \times Z_{2}(R E T) \\
& Z_{2} \text { (RET) } \times Z_{2} \text { (REt) } \\
& Z_{2} \text { (REt) } \times Z_{2} \text { (RTt) } \\
& Z_{2} \text { (RTt) } \times Z_{2} \text { (RE) } \\
& Z_{2} \text { (RE) } \times Z_{2} \text { (REt) } \\
& Z_{2} \text { (RETt) } \times Z_{2} \text { (Rt) } \\
& Z_{2}(\text { Rt }) \times Z_{2} \text { (RE) } \\
& Z_{2} \text { (RE) } \times Z_{2} \text { (RETt) }
\end{aligned}
$$$$
Z_{2}(\boldsymbol{R}) \times Z_{2}(E) \times Z_{2}(T) \times Z_{2}(t)
$$$$
Z_{2}(R T) \times Z_{2}(E) \times Z_{2}(T t)
$$$$
Z_{2}(R T) \times Z_{2}(E T) \times Z_{2}(T t)
$$$$
=Z_{2}(E) \times Z_{2}(R T t)
$$$$
=Z_{2}(R E T) \times Z_{2}(R T t)
$$$$
=Z_{2}(\boldsymbol{R E T}) \times Z_{2}(\boldsymbol{t})
$$$$
=\boldsymbol{Z}_{2}(\boldsymbol{E}) \times \boldsymbol{Z}_{2}(\boldsymbol{R t})
$$$$
=\boldsymbol{Z}_{2}(\boldsymbol{R E T}) \times \boldsymbol{Z}_{2}(\boldsymbol{R t})
$$$$
=Z_{2}(\text { RET }) \times Z_{2}(T t)
$$$$
=Z_{2}(E T) \times Z_{2}(R T t)
$$$$
=Z_{2}(R E) \times Z_{2}(R T t)
$$$$
=Z_{2}(R E) \times Z_{2}(t)
$$$$
=Z_{2}(E T) \times Z_{2}(R t)
$$$$
=Z_{2}(\boldsymbol{R E}) \times Z_{2}(\boldsymbol{R t})
$$$$
=Z_{2}(R E) \times Z_{2}(T t)
$$

Level IV

$Z_{2}(R T)$

$Z_{2}(R E)$

$Z_{2}$ (RET)

$Z_{2}(E T)$

$Z_{2}(E)$

$Z_{2}$ (REt)

$Z_{2}$ (RETt)

$Z_{2}(E T t)$

$Z_{2}$ (Et)

$Z_{2}(\boldsymbol{R t})$

$Z_{2}$ (RTt)

$Z_{2}(T t)$

$Z_{2}(\boldsymbol{t})$

Level $\mathrm{V}$
$1 x$

$1 \beta$

$2 x$

$2 \beta$

$1 \chi-2 \alpha$ interaction: A

$1 \beta-2 \beta$ interaction

$1 \alpha-2 \beta$ interaction: MP

$1 \beta-2 \alpha$ interaction

$1 x-1 \beta$ interaction

$2 x-2 \beta$ interaction

$1 \alpha$-crowncenter: nonsymm. $1 \alpha$

$1 \beta$-crowncenter

$2 \alpha$-crowncenter: $\mathrm{D}$

$2 \beta$-crowncenter

l $\alpha$-crown

$1 \alpha$-crown

$1 \alpha$-crown: $\mathrm{C}$

$1 \beta$-crown

$1 \beta$-crown

$1 \beta$-crown

$2 \alpha$-crown

$2 \alpha$-crown

$2 \alpha$-crown: nonsymm. $2 \alpha$

$2 \beta$-crown

$2 \beta$-crown

$2 \beta$-crown

center: ML

Axis 1

Axis 1

Axis 1

Axis 1

Axis 2

Axis 2

Axis 2

Axis 2

Axis 3

Axis 3: B2

Axis 3

Axis 3: Bl

1

Note: The generators of the groups (except at levels IV and V) can be chosen in various ways. The notation in the first column is the most elegant one from a theoretical point of view (as explained in the appendix) and the alternative notation, where necessary, anticipates the inclusion of dissipation as in Section 3. 


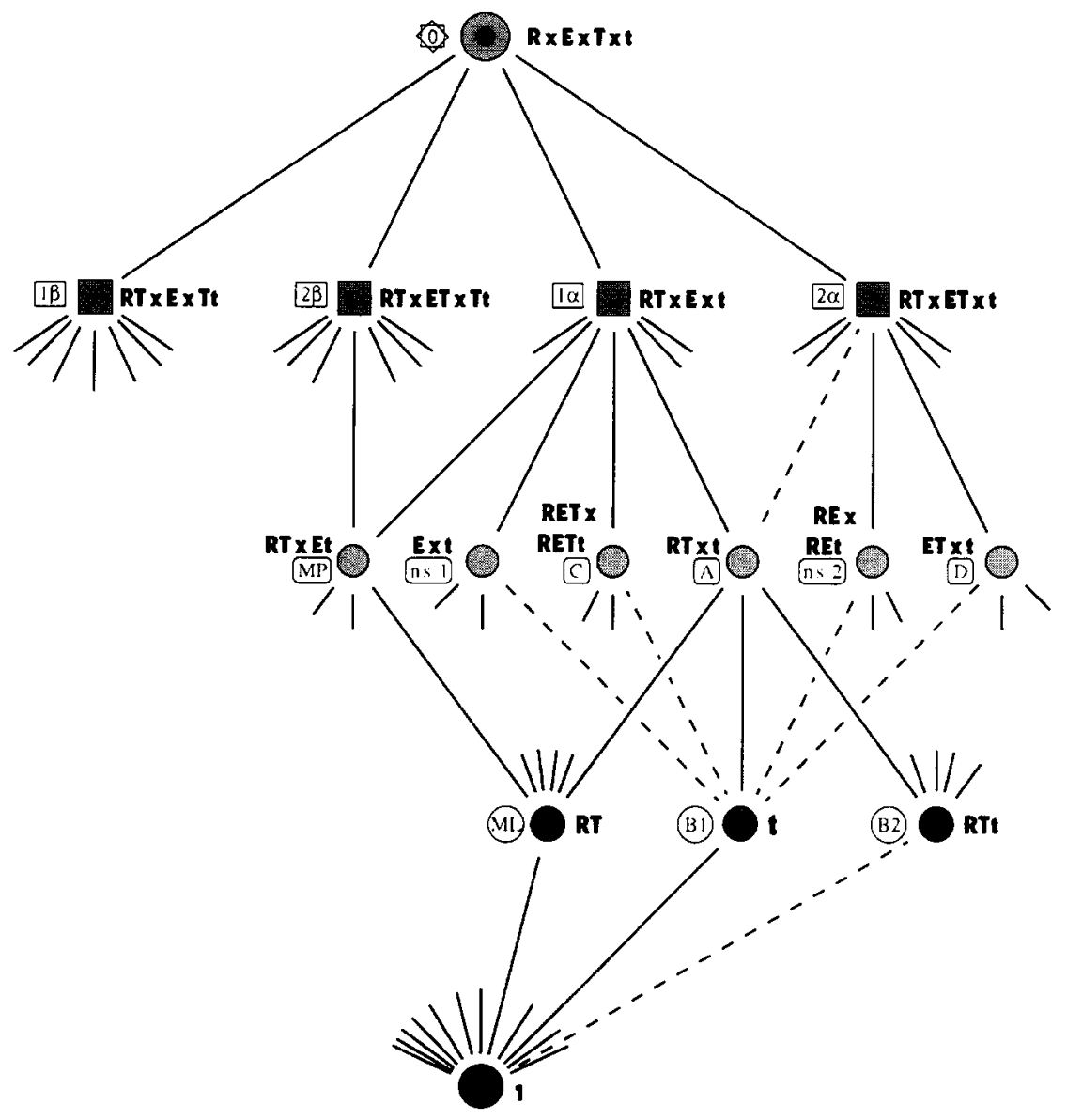

Fig. 3. The part of the Hamiltonian isotropy lattice corresponding to the observed oscillations of period $2 T$. The solid lines represent symmetry breaking bifurcations that have actually been observed in practice, the dashed lines correspond with symmetry breakings that are allowed in principle but have never been observed. The short lines indicate connections to groups associated with non-observed motions; these groups can be found in the pictures of the complete lattice in the appendix, see Figs. 12a-d.

15 groups of Fig. 3, covering the levels I, II and V completely but the levels III and IV only (very) partially. The vertices in this figure correspond to the observed motions (and their respective symmetry groups) and are labeled by their names as given in Refs. [ 1 3 ]. The solid lines indicate the observed mother-daughter connections, which take place via symmetry breaking bifurcations. There are also dashed lines in the figure; these indicate mother-daughter connections that are allowed in principle but have not been observed. For the sake of completeness, the short lines represent symmetry breaking bifurcations to motions that are quite possible from a group theoretical point of view but were never found within the investigated range of driving parameters [1-3]; in the appendix all the blanks associated with these nonobserved motions will be filled in. 
$2 \beta$

RTXETXTt

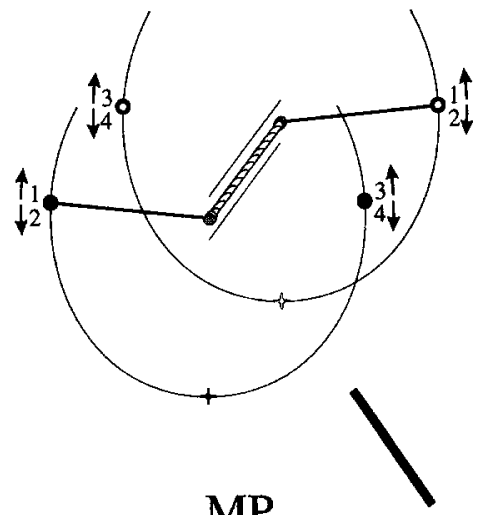

$1 \alpha$

\section{RTXEx!}
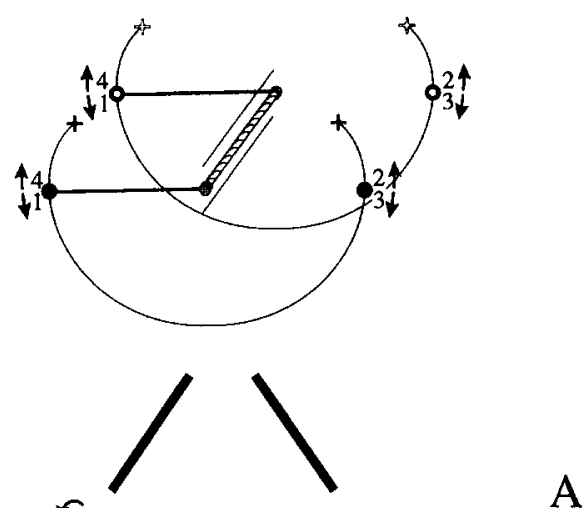

RTXEt
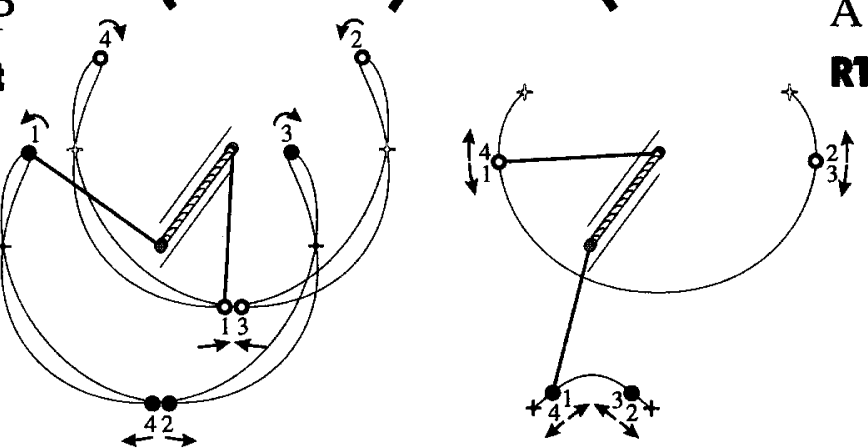

A

ML
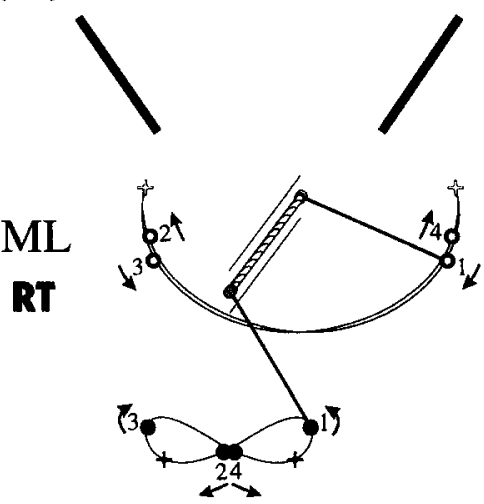

RT $x$ :

Fig. 4. The part of the Hamiltonian isotropy lattice corresponding to the observed mode interactions, being a small subset of Fig. 3 (which in turn is a small subset of the complete lattice given in the appendix). Apart from the group theoretical structure and the names of the various motion types (as in Fig. 3) we have also depicted what they look like in the two-pendulum system. 
Let us now consider the observed mode interactions in somewhat more detail. In Fig. 4 we have reproduced the (remarkably small) part of the lattice associated with this, together with real-life pictures of the oscillations involved. In these pictures the numbered spheres mark the position of the pendulums every time the bar of suspension passes its middle position; at the instants 1, 3 the bar is moving upwards and at 2, 4 it is moving downwards. The crosses signal the moments the bar of suspension goes through its lowest position; this moment was used in Refs. [1-3] for stroboscopic sampling.

Looking at Fig. 4 we see that the MP-motion is clearly some 'mixture' of $1 \alpha$ and $2 \beta$, and in the same way ML is a mix of MP and A. That is, the MP-motion looks remarkably like a linear superposition of the pictures of $1 \alpha$ and $2 \beta$, and ML resembles a superposition of MP and A; this is in fact the reason why we call them 'mixed' modes. Nevertheless, this interpretation must be taken with a grain of salt, since in a nonlinear system the linear superposition of two solutions does not generate a third one; or, put otherwise, the mixed motions are not (linearly) decomposable into constituent motions. Moreover, such an interpretation would suggest that a mixed motion can only be stable when both of its mothers are stable, but nothing could be further from the truth $[2,3,16]$.

Fig. 4 also illustrates how the various symmetries present themselves in the pendulum oscillations. Whenever there is $\boldsymbol{R} \boldsymbol{T}$ symmetry the trajectories of both pendulums are mirror symmetrical with respect to a vertical plane through the bar of suspension. An $\boldsymbol{E}$ symmetry means that the trajectories of the two pendulums are identical. (If the $\boldsymbol{E}$ symmetry appears on its own there is a direct point-to-point correspondence between the trajectories, while in the case of $\boldsymbol{E} \boldsymbol{T}$ or $\boldsymbol{E} \boldsymbol{t}$ the correspondence involves an extra temporal operation). The presence of the time reversal symmetry $\boldsymbol{t}$, pure or in combination with the other temporal symmetry $\boldsymbol{T}$, implies that the pendulums 'turn in their tracks'. They then trace out a single line in space; in the absence of $t$ and $T t$ the forward and backward swings are not identical and the trajectories show loop-like structures.

\section{The dissipative isotropy lattice}

The isotropy lattice of the dissipative system is much simpler than its Hamiltonian counterpart. This is a consequence of the absence of the time reversal symmetry $\boldsymbol{t}$, which greatly reduces the number of vertices in the lattice and even cuts away a whole level. To be specific, the lattice now has four levels:

level I : $Z_{2} \times Z_{2} \times Z_{2}$, with 8 elements per group,

level II : $Z_{2} \times Z_{2}, \quad$ with 4 elements per group,

level III : $Z_{2}$, with 2 elements per group ,

level IV : 1 ,

with 1 element (the identity). 
Table 2

The groups of the dissipative isotropy lattice

\begin{tabular}{ll}
\hline Level I & \\
$Z_{2}(R) \times Z_{2}(E) \times Z_{2}(T)$ & \\
Level II & $1 \alpha, 1 \beta$ \\
$Z_{2}(R T) \times Z_{2}(E)$ & $2 \alpha, 2 \beta$ \\
$Z_{2}(R T) \times Z_{2}(E T)$ & \\
Level III & C \\
$Z_{2}(R E T)$ & Nonsymmetrical $1 \alpha$ \\
$Z_{2}(E)$ & MP, A \\
$Z_{2}(R T)$ & Nonsymmetrical $2 \alpha$ \\
$Z_{2}(R E)$ & D \\
$Z_{2}(E T)$ & \\
Level IV & \\
$\mathbf{1}$ & B \\
\hline
\end{tabular}

Note: The group structures follow directly from those in Table 1 by omitting the generator containing $t$, for the 12 groups in Table 1 that are indicated by two alternative notations the second one has to be used.

Level I consists of a single vertex, representing (as before) the 0-motion. It now has only 2 daughters in the second level since, from a group theoretical point of view, there is no distinction anymore between the level II vertices formerly labeled $\alpha$ and $\beta$. The tetrahedral structure of the Hamiltonian lattice (see appendix) thus collapses already at the first stage; indeed, we will not be needing three dimensions to adequately display the complete dissipative isotropy lattice. Both level II vertices have three daughters in the third level. Since they have one common daughter, the $Z_{2}(R T)$ group, the number of level III vertices is equal to $6-1=5$. This number can also be calculated as follows: 8 (the number of elements in the original $Z_{2} \times Z_{2} \times Z_{2}$ group) minus 2 (the 'absent' vertices $\boldsymbol{R}$ and $\boldsymbol{T}$ ) minus 1 (the identity 1). A third way to find this number is by inspection of the Hamiltonian fourth level, which contains 13 vertices positioned on three orthogonal axes, see Fig. 12c in the appendix. The vertices on two of these axes contain $\boldsymbol{t}$ and hence disappear from the picture, and the remaining 5 vertices are the level III vertices of the dissipative picture. The five vertices of level III all have one and the same daughter, namely the identity 1; this single vertex constitutes the fourth and last level of the lattice. Adding up, the total number of vertices over the four levels equals $1+2+5+1=9$; they have been tabulated in Table 2 .

The 9 groups have been arranged in the form of a graph in Fig. 5. The level I vertex is positioned at the top of the hierarchy, and labeled in the same shorthand notation as in the previous section. The level II vertices are symbolized by squares, and the level III vertices are displayed as circles. The solid lines symbolize mother-daughter connections and indicate the allowed symmetry breaking bifurcations. The lattice shows that mode interaction between the 1 - and 2 -motions is possible via an interaction mode with group $Z_{2}(R T)$. In practice only the $1 \alpha$ - and the $2 \beta$-motions have actually been observed to interact via the MP-motion. Symbolically, this interaction is expressed as follows:

$$
2 \beta \rightarrow \mathrm{MP} \leftarrow 1 \alpha,
$$




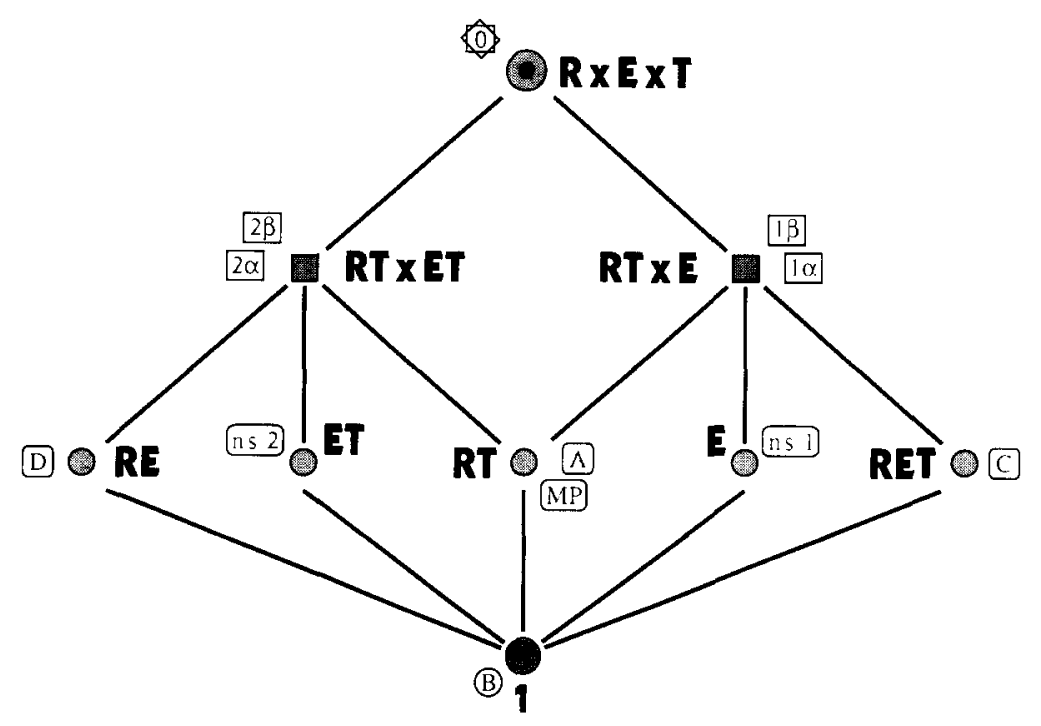

Fig. 5. The dissipative isotropy lattice.

where the arrows stand for symmetry breaking bifurcations. Other mode interactions which according to the isotropy would be equally possible, such as $2 \alpha \rightarrow \mathrm{A} \leftarrow 1 \alpha$, are not observed in practice; this is to say, the bifurcation from $1 \alpha$ to $\mathrm{A}$ is quite common (and important) but a bifurcation from $2 \alpha$ to $\mathrm{A}$ has never been witnessed in the parameter ranges investigated in Refs. [1-3].

It should be noted that one vertex may correspond with two or more different (i.e., physically distinguishable) types of motion. For instance, the $1 \alpha$-and $1 \beta$-motions have the same symmetry properties but they are separated by a saddle-node bifurcation and hence can be distinguished from each other; the same holds for the MP- and A-motions. In fact, in our experience, all the motions which occupied different vertices in the Hamiltonian lattice but collapse onto the same vertex in the dissipative lattice (for instance, the pairs $1 \alpha-1 \beta$ or A-MP) are still separated by a saddle node bifurcation. In this context it is illustrative to see how the Hamiltonian picture of mode interactions (Fig. 4) is affected. Its dissipative counterpart is shown in Fig. 6, where the dashed line between A and MP indicates the saddle-node bifurcation; it takes, so to speak, the place of the former ML-motion. Where in the Hamiltonian system one would have a bifurcation sequence $1 \alpha \rightarrow \mathrm{A} \rightarrow \mathrm{ML} \leftarrow \mathrm{MP} \leftarrow 2 \beta$, one now gets

$$
1 \alpha \rightarrow \mathrm{A} \stackrel{\mathrm{SN}}{\leftrightarrow} \mathrm{MP} \leftarrow 2 \beta,
$$

with the symbol $\stackrel{\mathrm{SN}}{\leftrightarrow}$ denoting the saddle-node bifurcation in which $\mathrm{A}$ and $\mathrm{MP}$ are created together.

As a side remark, it is interesting to note that the absence of time-reversal symmetry implies that in the dissipative system all motions show loop-like structures, as opposed to what we saw in the Hamiltonian case. 


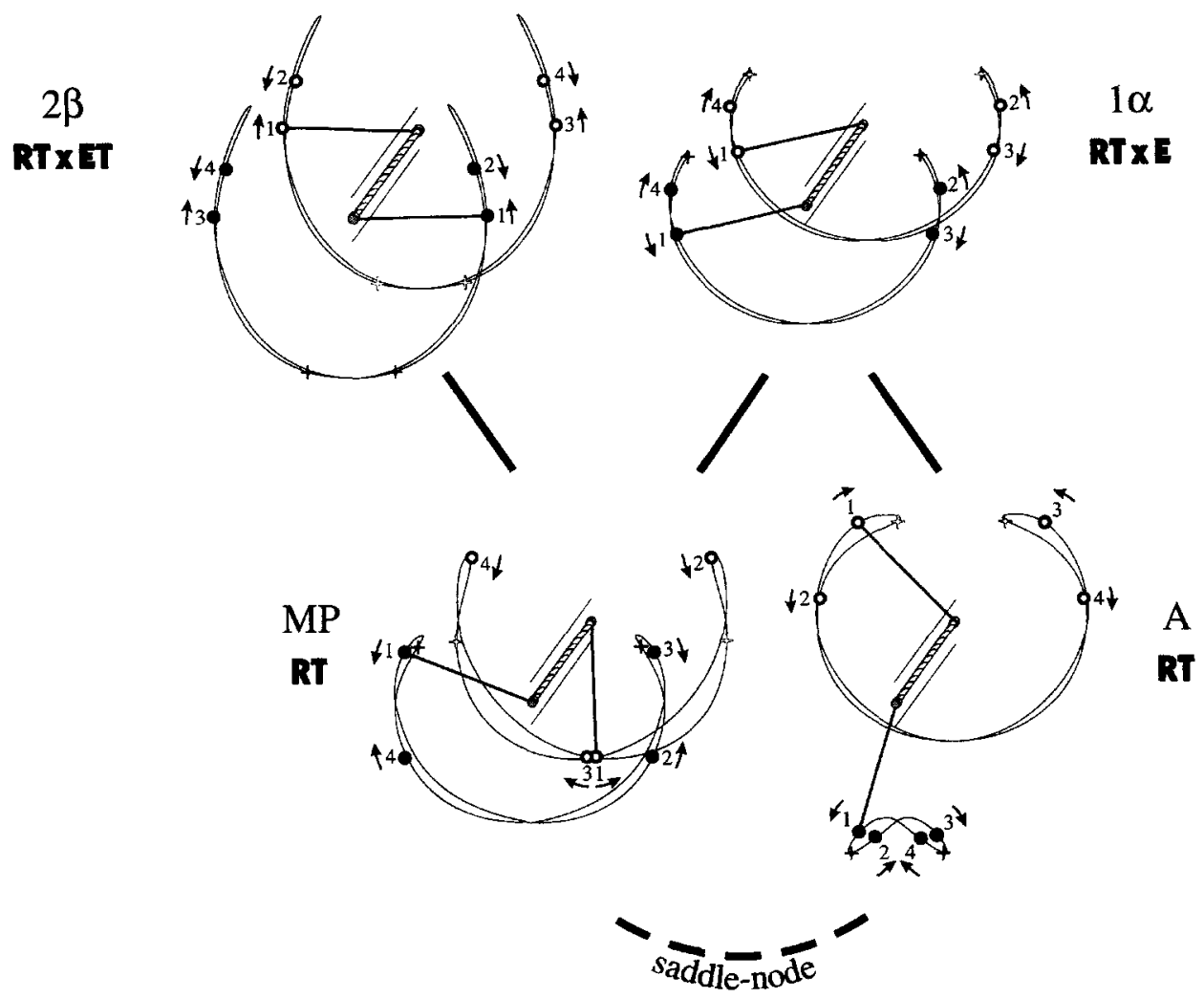

Fig. 6. The dissipative version of Fig. 4, showing the part of the isotropy lattice where the mode interaction takes place. The dashed line, representing the saddle-node bifurcation between the A- and MP-motion, has taken the place of the (Hamiltonian) ML-motion.

\section{Escape from the isotropy lattice}

\subsection{Bifurcations affecting the periodicity}

The bifurcational life of the oscillations originating, directly or indirectly, from the main resonance tongues is largely captured by the isotropy lattices discussed in the previous two sections. That is to say, the most important motions in the system all lie within the lattice (they have period $2 T$ ), the transitions from the one to the other taking place via symmetry breaking bifurcations. The system could be pulled out of the lattice by a period doubling or a Hopf bifurcation, since these affect the periodicity and generate motions with periods other than $2 T$. However, these types of bifurcation are relatively rare, since they can only occur for motions that are void of certain symmetries. To be specific, we will show in Section 4.2 that period doubling of a motion is (generically) impossible when it possesses a combination of the time translation symmetry $\boldsymbol{T}$ and a pure spatial symmetry ( $\boldsymbol{R}, \boldsymbol{E}$ or $\boldsymbol{R E})$. Subsequently, in 
Section 4.3, we show that the presence of a pure spatial symmetry prohibits the motion from undergoing a Hopf bifurcation.

The results derived in this section do not stand alone. In fact, bifurcations in the presence of symmetry form an active area of research; the interested reader can consult Ref. [15].

\subsection{Period doubling bifurcations}

Let us begin by constructing a stroboscopic map $\boldsymbol{M}$ from the equations of motion (Eq. (1.1)), by sampling the angles and angular velocities of the pendulums every time the bar of suspension goes through its lowest point. A motion $\bar{\theta}(t)$ with twice the period of the driving will show up as a fixed point of the second iterate of $\boldsymbol{M}$ :

$$
\bar{\theta}(t)=\boldsymbol{M}(\boldsymbol{M}(\bar{\theta}(t))) \equiv \boldsymbol{M}^{(2)}(\bar{\theta}(t)),
$$

for all values of $t$. The stability of $\bar{\theta}(t)$ is governed by the eigenvalues of the Jacobian matrix of the twice iterated stroboscopic map, defined by

$$
\left.\boldsymbol{L}^{(2)}\right|_{\bar{\phi}(t)}=\left.\frac{\partial \boldsymbol{M}^{(2)}}{\partial \theta}\right|_{\bar{\theta}(t)}=\left.\left.\boldsymbol{L}\right|_{\bar{\phi}(t+T)} \boldsymbol{L}\right|_{\bar{\partial}(t)}=\left.\left.\boldsymbol{L}\right|_{\bar{\tau} \bar{\phi}(t)} \boldsymbol{L}\right|_{\bar{\phi}(t)}
$$

The symplectic character of Hamiltonian systems guarantees $[17,18]$ that $\boldsymbol{M}$ is a symplectic map. ${ }^{4}$ This means that the Jacobian matrix of $\boldsymbol{M}$ is symplectic with respect to the standard symplectic matrix $\boldsymbol{J}$, i.e.,

$$
\boldsymbol{L}^{T} \boldsymbol{J}=\boldsymbol{J} \quad \text { with } \boldsymbol{J}=\left[\begin{array}{cc}
\emptyset & \boldsymbol{I}_{2} \\
-\boldsymbol{I}_{2} & \emptyset
\end{array}\right]
$$

Here $I_{2}$ denotes the $2 \times 2$ identity matrix. The $Z_{2}$-symmetries in our pendulum system can be expressed as symplectic matrices with respect to the same matrix $\boldsymbol{J}$ [17]. The product of two symplectic matrices is again a symplectic matrix, and its eigenvalues appear either as complex pairs on the unit circle $(\lambda, \bar{\lambda}$, with $|\lambda|=1)$, as real pairs $\left(\lambda, \lambda^{-1}\right)$, or as quadruplets $\left(\lambda, \bar{\lambda}, \lambda^{-1}, \bar{\lambda}^{-1}\right)$.

Suppose now that $\bar{\theta}(t)$ possesses a combination of $\boldsymbol{T}$ and a spatial symmetry, for example ET. In that case it cannot undergo a period doubling, i.e., when an eigenvalue pair arrives at -1 it will, generically, stay on the unit circle. To prove this we first rewrite the $\boldsymbol{E} \boldsymbol{T}$ symmetry, $\boldsymbol{E} \overline{\boldsymbol{\theta}}(t)=\bar{\theta}(t)$, as follows:

$$
\boldsymbol{T} \bar{\theta}(t)=\boldsymbol{E}^{-1} \bar{\theta}(t)=\boldsymbol{E} \bar{\theta}(t),
$$

or $\overline{\boldsymbol{T}} \overline{\boldsymbol{\theta}}=\boldsymbol{E} \bar{\theta}$ for short. Here we have used the fact that $\boldsymbol{E}$ is its own inverse. Eq. (4.2) then tells us that the stability of $\bar{\theta}(t)$ is governed by the eigenvalues of

$$
\left.\boldsymbol{L}^{(2)}\right|_{\bar{\theta}}=\left.\left.\boldsymbol{L}\right|_{\boldsymbol{T} \bar{\theta}} \boldsymbol{L}\right|_{\bar{\theta}}=\left.\left.\boldsymbol{L}\right|_{\boldsymbol{E} \overline{\bar{\theta}}} \boldsymbol{L}\right|_{\bar{\theta}}
$$

\footnotetext{
${ }^{4}$ In the present analysis we treat the Hamiltonian case. If one includes viscous dissipation the map $\boldsymbol{M}$ becomes semi-symplectic [27] and the argument proceeds analogously.
} 

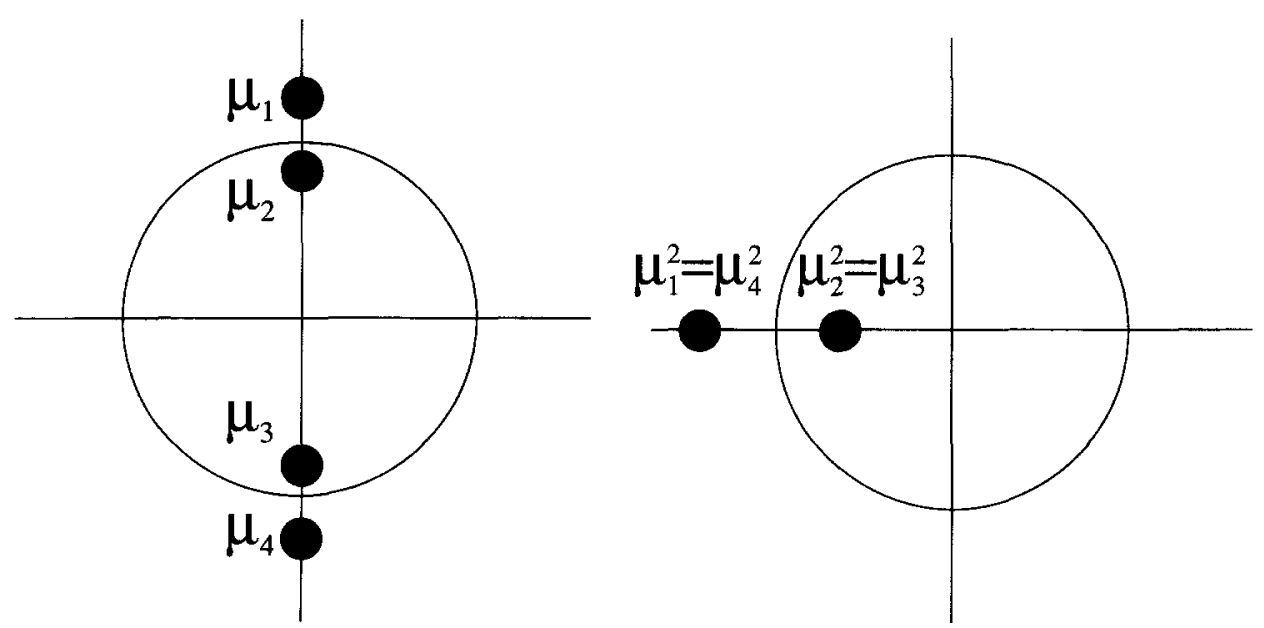

Fig. 7. The eigenvalues of a matrix which is the square of a symplectic matrix can only lie on the negative real axis if they are degenerate, i.e., if the eigenvalues of the 'square-root' symplectic matrix form a quadruplet on the imaginary axis.

Now, since $\boldsymbol{E}$ is a symmetry of the differential equations (i.e., of the pendulum system) it is also a symmetry of the stroboscopic map:

$$
\boldsymbol{M}(\boldsymbol{E}(\theta(t)))=\boldsymbol{E}(\theta(t+T))=\boldsymbol{E}(\boldsymbol{M}(\theta(t))) .
$$

Differentiating with respect to $\theta$ and evaluating at $\theta=\bar{\theta}$ yields for the left-hand side

$$
\left.\frac{\partial \boldsymbol{M}(\boldsymbol{E} \theta)}{\partial \theta}\right|_{\bar{\theta}}=\left.\frac{\partial \boldsymbol{M}(\boldsymbol{E} \theta)}{\partial(\boldsymbol{E} \theta)} \frac{\partial(\boldsymbol{E} \theta)}{\partial \theta}\right|_{\bar{\theta}}=\left.\boldsymbol{L}\right|_{\boldsymbol{E} \bar{\theta}} \boldsymbol{E},
$$

and for the right-hand side

$$
\left.\frac{\partial \boldsymbol{E} \boldsymbol{M}(\theta)}{\partial \theta}\right|_{\bar{\theta}}=\left.\frac{\partial \boldsymbol{E} \boldsymbol{M}(\theta)}{\partial \boldsymbol{M}(\theta)} \frac{\partial \boldsymbol{M}(\theta)}{\partial \theta}\right|_{\bar{\theta}}=\left.\boldsymbol{E} \boldsymbol{L}\right|_{\bar{\theta}} .
$$

Thus we obtain

$$
\left.\boldsymbol{L}\right|_{\boldsymbol{E} \overline{\hat{\theta}}} \boldsymbol{E}=\left.\boldsymbol{E} \boldsymbol{L}\right|_{\bar{\theta}} \quad \text { or }\left.\quad \boldsymbol{L}\right|_{\boldsymbol{E} \overline{\hat{\theta}}}=\left.\boldsymbol{E} \boldsymbol{L}\right|_{\bar{\theta}} \boldsymbol{E},
$$

and with this we can write Eq. (4.5) as

$$
\left.L^{(2)}\right|_{\bar{\theta}}=\left.\left.L\right|_{E \bar{\theta}} L\right|_{\bar{\theta}}=\left.\left.E L\right|_{\bar{\theta}} E L\right|_{\bar{\theta}}
$$

That is, the Jacobian matrix of the second iterate of $\boldsymbol{M}$ is equal to the square of the symplectic matrix $\left.E L\right|_{\bar{\theta}}$. (Similar quadratic behavior has been described in Refs. [1,19].) So two eigenvalues of $\left.\boldsymbol{L}^{(2)}\right|_{\bar{\theta}}$ can lie on the negative real axis only if they are degenerate, i.e., if $\boldsymbol{E} \boldsymbol{L}_{\bar{\phi}}$ has a quadruplet of eigenvalues on the imaginary axis, as in Fig. 7. This can be the case in isolated points in the $(A, \Omega)$-plane but not along whole lines. 
Thus, there can be no period doubling bifurcation line for a motion with $E T$ symmetry and, more generally, it follows that such lines are ruled out for any motion that has a combination of the $\boldsymbol{T}$ symmetry with a spatial symmetry; that is, for all but four of the observed motions in the Hamiltonian isotropy lattice. The only observed motions that can (and do) undergo a period doubling bifurcation are the non-symmetrical $1 \alpha(\boldsymbol{E}, \boldsymbol{t}, \boldsymbol{E} \boldsymbol{t}, \mathbf{1})$, the nonsymmetrical $2 \alpha(\boldsymbol{R E}, \boldsymbol{t}, \boldsymbol{R E} \boldsymbol{t}, \mathbf{1})$ and the mixed motions B1 and B2 ( $t, 1$ and $R T t, 1$, respectively). So indeed, escape from the lattice via period doubling is seen to be a relatively rare phenomenon. ${ }^{5}$ This remains true in the presence of viscous damping; in that case the nonsymmetrical $1 \alpha$ - and $2 \alpha$-motions ( $\boldsymbol{E}, \mathbf{1}$ and $\boldsymbol{R E}, \mathbf{1}$, respectively) and the B-motion (1) are the only three observed motions that undergo period doubling.

\subsection{Hopf bifurcations}

A second way to escape from the isotropy lattice is by means of a Hopf bifurcation. In the context of mappings this bifurcation is also known as the Neimark-Sacker bifurcation [20] and describes the birth of a limit cycle, introducing quasiperiodic behavior into the system (whereas in the context of flows a Hopf bifurcation creates periodic behavior). The bifurcation is related to the birth of an eigenvalue quadruplet of the stroboscopic map $\boldsymbol{M}$; the actual bifurcation takes place at the moment when two of the four quadruplet-eigenvalues move outside the unit circle. In a Hamiltonian system these two moments (the birth of the quadruplet and the Hopf bifurcation) coincide, but in the presence of dissipation the birth precedes the bifurcation. Now, the eigenvalues corresponding to a motion confined to a plane in stroboscopic phase space can never form a quadruplet, since for such motions the eigenvalues always come in pairs and lie either on the real axis or on the unit circle (the latter being replaced by the reduced circle [2] if dissipation is present); these motions can therefore not undergo Hopf bifurcations. In this subsection we will show that such motions can be recognized by the presence of a pure spatial symmetry.

Consider a motion $\bar{\theta}(t)$ that possesses, for instance, the $\boldsymbol{R} \boldsymbol{E}$ symmetry. In matrix language this is expressed as:

$$
\boldsymbol{X}_{\mathbf{R E}, \vartheta} \bar{\theta}(t)=\bar{\theta}(t)
$$

or, more explicitly,

$$
\left[\begin{array}{cccc}
0 & 0 & -1 & 0 \\
0 & 0 & 0 & -1 \\
-1 & 0 & 0 & 0 \\
0 & -1 & 0 & 0
\end{array}\right]\left(\begin{array}{c}
\bar{\vartheta}_{1}(t) \\
\overline{\bar{\vartheta}}_{1}(t) \\
\bar{\vartheta}_{2}(t) \\
\dot{\bar{\vartheta}}_{2}(t)
\end{array}\right)=\left(\begin{array}{c}
-\bar{\vartheta}_{2}(t) \\
-\dot{\bar{\vartheta}}_{2}(t) \\
-\bar{\vartheta}_{1}(t) \\
-\overline{\bar{\vartheta}}_{1}(t)
\end{array}\right)=\left(\begin{array}{c}
\bar{\vartheta}_{1}(t) \\
\dot{\bar{\vartheta}}_{1}(t) \\
\bar{\vartheta}_{2}(t) \\
\overline{\bar{\vartheta}}_{2}(t)
\end{array}\right),
$$

\footnotetext{
${ }^{5}$ If we do not restrict ourselves to the observed motions but consider the complete lattice, we find that none of the 4 level II vertices, eight of the 22 level III vertices and ten of the 13 level IV vertices admit period doubling bifurcations.
} 
where $\boldsymbol{X}_{\boldsymbol{R E}, \vartheta}$ stands for the matrix representation of the $\boldsymbol{R E}$ symmetry in the $\vartheta$-coordinates (For convenience we have written the transformation only for the stroboscopic phase space coordinates, i.e, not for the time coordinate, since the latter is unaffected). Note that $X_{\mathbf{R E}, \vartheta}$ has two eigenvalues equal to -1 (and two equal to 1 ); we shall show that this fact enables us to describe the motion under consideration using one degree of freedom less. To this end we bring $\boldsymbol{X}_{\boldsymbol{R E}, \vartheta}$ into diagonal form, using the coordinate transformation given by the inverse of the matrix of eigenvectors of $\boldsymbol{X}_{\mathbf{R E}, \vartheta}$, namely:

$$
\boldsymbol{V}_{\boldsymbol{R E}, \vartheta}^{-1}=\left[\begin{array}{cccc}
\frac{1}{2} \sqrt{2} & 0 & \frac{1}{2} \sqrt{2} & 0 \\
-\frac{1}{2} \sqrt{2} & 0 & \frac{1}{2} \sqrt{2} & 0 \\
0 & \frac{1}{2} \sqrt{2} & 0 & \frac{1}{2} \sqrt{2} \\
0 & -\frac{1}{2} \sqrt{2} & 0 & \frac{1}{2} \sqrt{2}
\end{array}\right] .
$$

The new coordinates (the 'natural' coordinates for a motion with $\boldsymbol{R} \boldsymbol{E}$ symmetry) are given by

$$
\boldsymbol{V}_{\boldsymbol{R E}, \vartheta}^{-1}\left(\begin{array}{c}
\vartheta_{1} \\
\dot{\vartheta}_{1} \\
\vartheta_{2} \\
\dot{\vartheta}_{2}
\end{array}\right)=\frac{1}{2} \sqrt{2}\left(\begin{array}{c}
\vartheta_{1}+\vartheta_{2} \\
-\vartheta_{1}+\vartheta_{2} \\
\dot{\vartheta}_{1}+\dot{\vartheta}_{2} \\
-\dot{\vartheta}_{1}+\dot{\vartheta}_{2}
\end{array}\right) \equiv\left(\begin{array}{c}
\phi_{1} \\
-\phi_{2} \\
\dot{\phi}_{1} \\
-\dot{\phi}_{2}
\end{array}\right) .
$$

These are not surprisingly, just the familiar normal coordinates for our pendulum system [1-3]. The matrix representation of the $\boldsymbol{R} \boldsymbol{E}$ symmetry in the new coordinates is found as follows:

$$
\boldsymbol{V}_{\boldsymbol{R E}, \vartheta} \boldsymbol{X}_{\boldsymbol{R E}, \vartheta} \boldsymbol{V}_{\boldsymbol{R}, \vartheta}^{-1}=\left[\begin{array}{cccc}
-1 & 0 & 0 & 0 \\
0 & 1 & 0 & 0 \\
0 & 0 & -1 & 0 \\
0 & 0 & 0 & 1
\end{array}\right] \equiv \boldsymbol{X}_{\mathrm{RE}, \Phi}
$$

and this is the diagonal matrix we were looking for. Now, the action of $\boldsymbol{R E}$ on the motion in the new coordinates is expressed as

$$
\boldsymbol{X}_{\mathrm{RE}, \Phi} \bar{\Phi}(t)=\bar{\Phi}(t)
$$

or, written more explicitly,

$$
\left[\begin{array}{cccc}
-1 & 0 & 0 & 0 \\
0 & 1 & 0 & 0 \\
0 & 0 & -1 & 0 \\
0 & 0 & 0 & -1
\end{array}\right]\left(\begin{array}{c}
\bar{\phi}_{1}(t) \\
-\bar{\phi}_{2}(t) \\
\dot{\bar{\phi}}_{1}(t) \\
-\bar{\phi}_{2}(t)
\end{array}\right)=\left(\begin{array}{c}
-\bar{\phi}_{1}(t) \\
-\bar{\phi}_{2}(t) \\
-\overline{\bar{\phi}}_{1}(t) \\
-\dot{\bar{\phi}}_{2}(t)
\end{array}\right)=\left(\begin{array}{c}
\bar{\phi}_{1}(t) \\
-\bar{\phi}_{2}(t) \\
\dot{\bar{\phi}}_{1}(t) \\
-\overline{\bar{\phi}}_{2}(t)
\end{array}\right)
$$

and from this we immediately see that $\bar{\phi}_{1}(t)=0$ as well as $\dot{\bar{\phi}}_{1}(t)=0$, for all $t$. Thus we conclude that any motion which possesses $\boldsymbol{R E}$ symmetry is confined to a plane in stroboscopic phase space (defined by $\phi_{1}=0$ and $\dot{\phi}_{1}=0$ ). This means that it cannot undergo a Hopf bifurcation. 
In effect, we have demonstrated that a motion with $\boldsymbol{R E}$ symmetry is a normal motion; ${ }^{6}$ indeed, it is a (symmetrical or nonsymmetrical) 2-motion and as a matter of fact we already knew [1] that this type of motion cannot undergo a Hopf bifurcation. The interesting new thing is of course that we have shown this by using the spatial symmetry of the motion, obtaining its natural coordinates as a bonus. The proof hinges on the fact that the matrix representation has two eigenvalues equal to -1 ; conveniently enough, all the spatial symmetries in our system have matrix representations with either two or four eigenvalues equal to -1 . (This number cannot be odd because we are dealing with linear $Z_{2}$-symmetries: the determinant of their matrices is equal to +1 , so the number of negative eigenvalues must be even.) Each pair of negative eigenvalues of a spatial symmetry effectively eliminates one degree of freedom (or, equivalently, two phase-space dimensions) from a motion. Both $\boldsymbol{E}$ and $\boldsymbol{R} \boldsymbol{E}$ have matrices with one pair of negative eigenvalues; the respective motions (1- and 2-motions) are accordingly confined to a plane in stroboscopic phase space. The matrix associated with the symmetry of reflection, $\boldsymbol{R}$, has two pairs of negative eigenvalues; the only motion with $\boldsymbol{R}$-symmetry, the downward equilibrium motion, is indeed confined to a point in stroboscopic phase space.

In the Hamiltonian isotropy lattice all this means that there is not a single motion in level I or II which admits a Hopf bifurcation. However, in level III there are 12 vertices (out of 22) and in level IV no less than 11 (out of 13) which correspond to motions that can undergo a Hopf bifurcation. Of all the observed motions in level III only the nonsymmetrical $1 \alpha$ - and $2 \alpha$-motions cannot give way to quasiperiodic behavior; the rest of the observed motions in level III and IV can. Thus, the escape from the lattice by means of a Hopf bifurcation can (and does) occur more often than escape via a period doubling. This remains true in the presence of viscous damping; also in that case the levels I and II are ruled out, but in the lower regions of the lattice only the nonsymmetrical 1- and 2-motions are not allowed to undergo a Hopf bifurcation.

In conclusion, we have shown that any escape from the isotropy lattice must necessarily occur below level II (the level of the pure modes). At level III escape is possible, but not from every vertex; moreover, when escape is allowed it is either via a Hopf or a period doubling bifurcation, but not in both ways. It is not before the fourth level that escape from the lattice becomes the rule rather than the exception; it is now possible from each vertex, and often in both ways. In this context, however, it should be noted that the vertices which survive dissipation (and are therefore the most important ones from a practical point of view) are exactly those which admit only one type of escape. All in all, the results in this section explain the observational fact that the dynamics of our two-pendulum system is largely captured by motions belonging to the period-2 isotropy lattice.

\footnotetext{
$\overline{{ }^{6} \text { The argument }}$ can also be used in reverse order to establish the spatial symmetries of any given normal mode.
} 


\section{Comparison with other systems}

In our previous papers we have regularly referred to other systems which exhibit mode interaction, but postponed a detailed comparison until we had a generic (rather than system-specific) picture of the phenomenon. The group theoretical approach provides us with such a picture and the time has finally come to carry out the comparison. We shall discuss two systems in particular, namely the compound pendulum of Skeldon and Mullin, and the Faraday experiment as performed by Ciliberto and Gollub. It should be noted that these systems have also been studied extensively, like our two-pendulum system, on the level of the equations of motion (for the compound pendulum one may consult Refs. [6,7], and for the Faraday experiment Refs. [21-26]); but in the present context we operate on the more abstract level of symmetry groups.

The compound pendulum of Skeldon and Mullin [6,7], sketched in Fig. 8, consists of two rods joined in such a way that they swing in perpendicular directions. The point of suspension is being moved up and down harmonically, so (like our own two pendulums) the system is parametrically driven. The group theoretical structure of the oscillations associated with the main resonance tongues is exactly the same as in our case; that is, the trivial motion from which all the others follow has (in the presence of dissipation) $Z_{2} \times Z_{2} \times Z_{2}$ symmetry. The first two $Z_{2}$ symmetries are reflections of the angles $\vartheta$ and $\phi, Z_{2}\left(\boldsymbol{R}_{\vartheta}\right)$ and $Z_{2}\left(\boldsymbol{R}_{\phi}\right)$, and the third one is the familiar time shift by one period of the driving, $Z_{2}(\boldsymbol{T})$ (again we restrict ourselves to $2 T$-periodic motions). The two pure modes that are born from the trivial motion are the $\phi$-mode, in which the upper rod hangs vertically downward and only the lower one is oscillating, and the $\vartheta$-mode, in which the whole contraption moves as one, i.e., the upper rod oscillates and the lower one follows suit, locked in its joint. The symmetry groups of these pure modes are $\boldsymbol{Z}_{2}\left(\boldsymbol{R}_{\vartheta}\right) \times \boldsymbol{Z}_{2}\left(\boldsymbol{R}_{\phi} \boldsymbol{T}\right)$ and $\boldsymbol{Z}_{2}\left(\boldsymbol{R}_{\vartheta} \boldsymbol{T}\right) \times \boldsymbol{Z}_{2}\left(\boldsymbol{R}_{\phi}\right)$, to be compared with

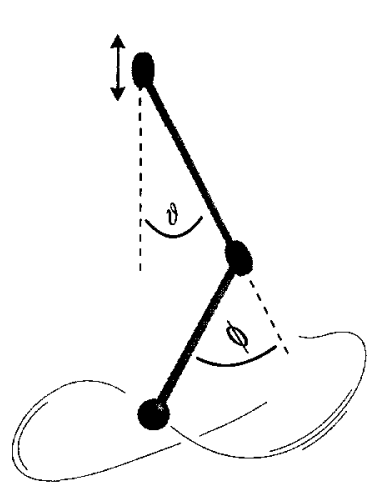

(a)

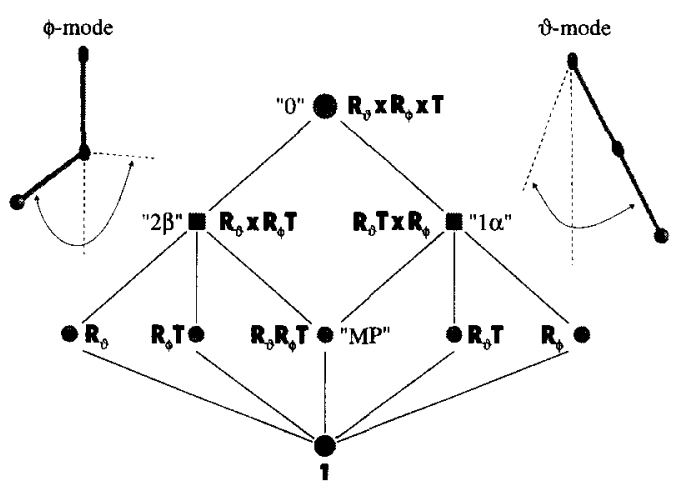

(b)

Fig. 8. The parametrically driven compound pendulum (after Refs. [6,7]), together with the corresponding (dissipative) isotropy lattice. 


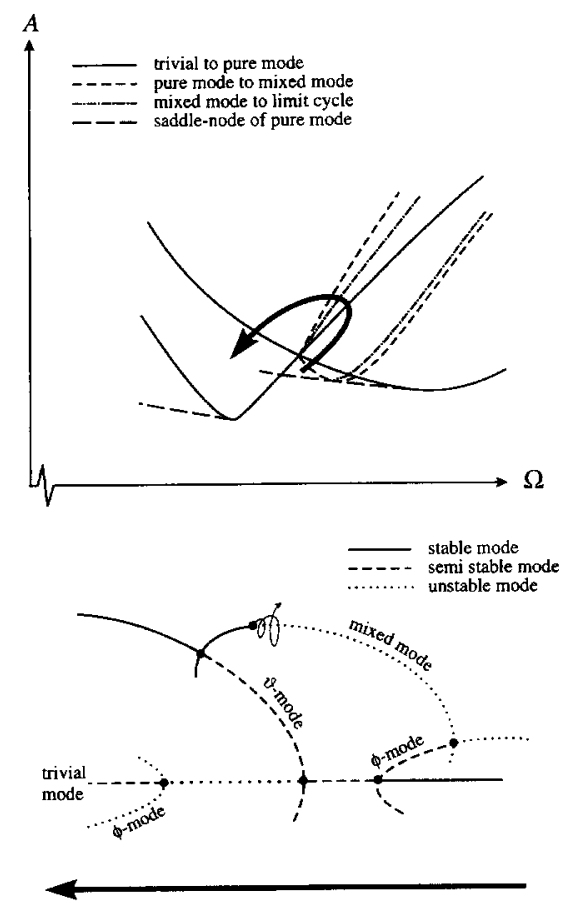

(a)

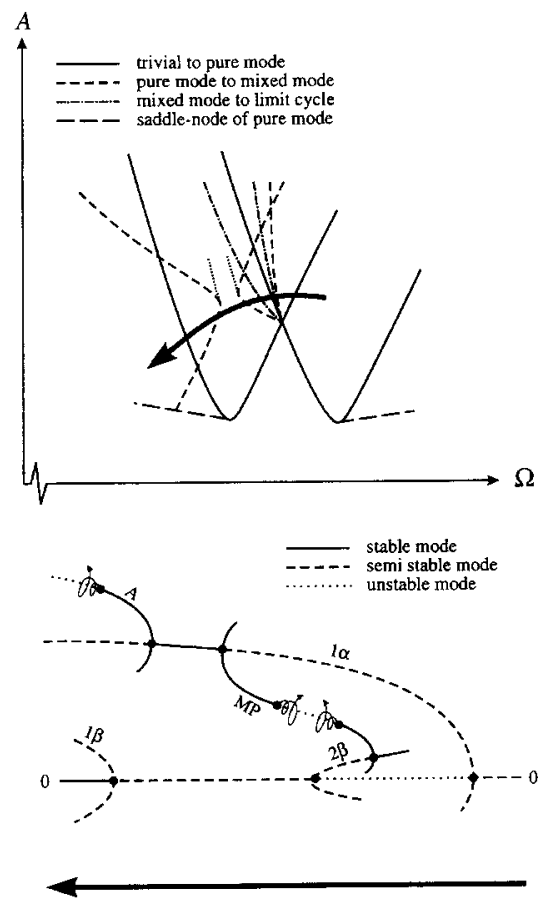

(b)

Fig. 9. The $(A, \Omega)$-plane, plus bifurcation diagrams along the arrow in the $(A, \Omega)$-plane, for (a) the compound pendulum; (b) our two-pendulum system.

the symmetrical 2- and 1-motions in our two-pendulum system. The mode interaction, between the counterparts of our $2 \beta$ - and $1 \alpha$-motion, is again established by the common daughter (corresponding to our MP-motion) with symmetry group $\boldsymbol{Z}_{2}\left(\boldsymbol{R}_{v} \boldsymbol{R}_{\phi} \boldsymbol{T}\right)$. The complete dissipative isotropy lattice is included in Fig. 8.

Despite the fact that the group theoretical structure is identical to that of our twopendulum system, the position of the mode interaction region (with respect to the intersection point of the resonance tongues) is quite different. In Fig. 9 we have reproduced the $(A, \Omega)$-planes of both systems side by side, together with the bifurcation diagrams associated with paths, indicated by the arrows, around the mode interaction points. The obvious similarities testify to the fact that the underlying mechanism of the mode interaction is the same in both cases; the discrepancies stem from differences in the nonlinearities of the systems. The coupling terms, for instance, differ considerably: in our case the pendulums are coupled via a torsion spring, while the coupling in the compound pendulum resides in the joint. It should not come as a surprise that such differences can have huge consequences, since in our own system we have already seen something similar when we included a (small) cubic term in the coupling [3]. Without this term, i.e., with a purely linear coupling, we hardly had any mode interaction at all. 


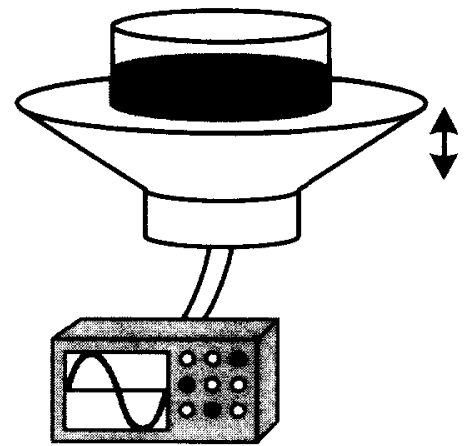

(a)

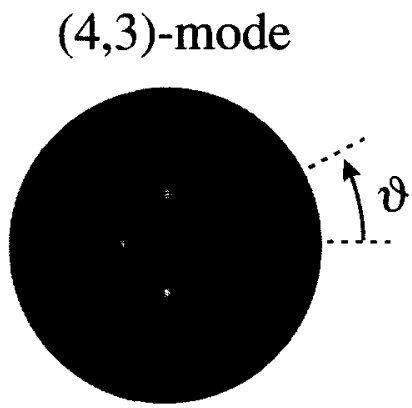

(b)

Fig. 10. (a) Schematic setup of the Faraday experiment. (b) The (4,3)-mode, represented by the surface plot of $J_{4}(r) \cdot \cos (4 \vartheta)$, with $J_{4}(r)$ a fourth-order Bessel function. The numbers indicate that the pattern has 4 maxima in the angular direction and $2(=3-1)$ nodes in the radial direction.

Another system with the same $Z_{2} \times Z_{2} \times Z_{2}$ structure is described in Refs. [ 8-10], where the mode interaction of cross-waves in a rectangular wave tank is investigated. Also in this case the location of the interaction region is greatly influenced by the specific nonlinearities in the system's equations of the motion.

The Faraday experiment, involving the wave patterns on the surface of a parametrically driven fluid container (see Fig. 10) has a different group structure. In the case of a circular container the spatial symmetry of the flat fluid surface is given by $O(2)$, the orthogonal group in two dimensions, consisting of all rotations around the centre of the container and all reflections in axes that pass through that centre. Crawford et al. [26] have made a detailed investigation of the symmetries of this system, with particular emphasis on the mode interaction between the surface patterns $(4,3)$ and $(7,2)$ as observed by Ciliberto and Gollub [4,5]; here we reproduce only the essence of their analysis.

The group structure of the trivial motion (the flat fluid surface that is being moved up and down) needed for a proper description of the mode interaction is $O(2) \times$ $Z_{2}(\boldsymbol{T})$. The subgroups corresponding to the $(7,2)$ and $(4,3)$ modes are $\tilde{D}_{14}(\boldsymbol{R},(2 \pi / 14) \boldsymbol{T})$ and $\tilde{D}_{8}(\boldsymbol{R},(2 \pi / 8) \boldsymbol{T})$, respectively [26]. Here $\tilde{D}_{2 l}(\boldsymbol{R},(2 \pi / 2 l) \boldsymbol{T})$ is the dihedral group generated by the reflection $\boldsymbol{R}$, given by $\vartheta \rightarrow-\vartheta$, and the element $(\pi / l) \boldsymbol{T}$, which represents a rotation over an angle $(\pi / l)$ combined with a time shift by one driving period. In Fig. 1la the corresponding symmetry lattice is presented; it is seen that, in this case, the pure modes have two common daughters, implying that there are two ways to interact. The difference between these two ways is rather subtle. The interaction mode with symmetry group $Z_{2}(\boldsymbol{R})$ is invariant under the (purely spatial) reflection $\vartheta \rightarrow-\vartheta$, while its sister with symmetry group $Z_{2}(\boldsymbol{R T})$ is left unchanged by the (spatio-temporal) operation of reflection combined with time translation. In the experiment of Ciliberto and Gollub it was not specified which of these two established the actual interaction between the two pure modes; in fact, only the Hopf bifurcated version of one of them 
(a)
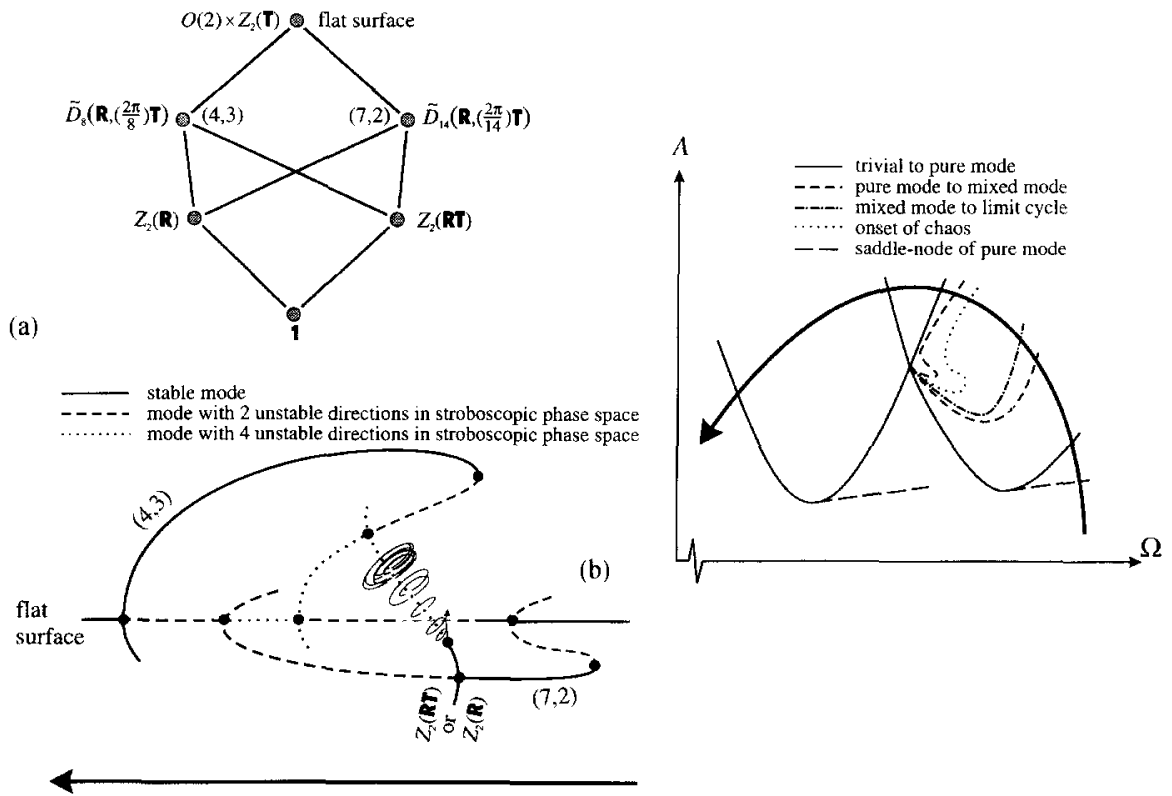

Fig. 11. (a) The isotropy lattice corresponding to the interaction between the pure modes $(7,2)$ and $(4,3)$ in the Faraday experiment (after Ref. [26]). (b) The $(A, \Omega)$-plane and the bifurcation diagram along the arrow in the $(A, \Omega)$-plane

was observed. This Hopf bifurcation introduces an extra periodicity into the motion, generating periodic amplitude oscillations, but does not affect the $Z_{2}$ symmetry [26]. The amplitude oscillations were found to subsequently undergo period doublings and eventually become chaotic; that is why the original paper by Ciliberto and Gollub was titled 'Pattern competition leads to chaos'.

In Fig. $11 \mathrm{~b}$ the neighborhood of the mode interaction point in the $(A, \Omega)$-plane is sketched, together with the bifurcation diagram along the arrow. It may be noted that a dashed line in this bifurcation diagram does not imply semi-stability, but rather that the corresponding mode has two unstable directions in stroboscopic phase space; this still leaves innumerable stable directions, since the number of degrees of freedom in the Faraday experiment is much larger than 2. Similarly, a dotted line does not imply full instability; it just indicates that there are four unstable directions.

In spite of a few conspicuous differences with respect to the two-pendulum picture (e.g., the fact that all the bifurcations from the trivial motion are now directed towards the right) it is obvious from Fig. $11 \mathrm{~b}$ that the mechanism for mode interaction between the two pure modes is the same: it is again established by a common daughter in the symmetry hierarchy. The analogy goes even further, in that the interaction mode is seen to undergo the same kind of transition to chaos, via a Hopf bifurcation and ensuing period doublings; but this is of course a less generic similarity, since it depends on the specific nonlinearities of the two systems and (as shown in Section 4) on the symmetries involved. 


\section{Summary and conclusions}

In this paper we bring our study of two coupled, parametrically driven pendulums to an end. The first three papers [1-3] give a detailed description of the specific dynamics of the oscillations arising, directly or indirectly, from the main tongues of parametric resonance. In the present paper we take a more general point of view and concentrate on the symmetries of the system rather than on (the specific form of) the equations of motion. It turns out that behind the multitude of bifurcation sequences observed in practice lies an overall structure that is determined by the symmetry of the system.

The symmetries relevant for the oscillations we are interested in, as discussed already in the previous papers, are associated with the operations of reflection $(\boldsymbol{R})$, exchange of pendulums $(\boldsymbol{E})$, time shift by one driving period $(\boldsymbol{T})$ and, in the Hamiltonian case, the operation of time reversal $(\boldsymbol{t})$. Since these operations are their own inverse (for $\boldsymbol{T}$ this means that we restrict ourselves to motions with twice the period of the driving) the relevant symmetry group of the dissipative system is of the form $Z_{2} \times Z_{2} \times Z_{2}$, and that of the Hamiltonian system $Z_{2} \times Z_{2} \times Z_{2} \times Z_{2}$. The trivial equilibrium is the only motion which possesses all the symmetries of this group; the oscillatory motions with period $2 T$ correspond with subgroups of $Z_{2} \times Z_{2} \times Z_{2}$ (or $Z_{2} \times Z_{2} \times Z_{2} \times Z_{2}$ ) and are organized in the so-called isotropy lattice. In the present paper we have constructed and interpreted this lattice; the Hamiltonian version is presented in Section 2 (and the appendix) and its dissipative counterpart in Section 3. The vertices in the isotropy lattices represent the subgroups and the lines connecting them indicate the allowed symmetry breaking bifurcations.

The mode interactions in the two-pendulum system can readily be recognized in the isotropy lattices. Not all of the interactions suggested by the lattices are necessarily observed in practice, though. The Hamiltonian lattice is full of possible mode interactions, but only two have been observed in the two-pendulum system. The first one is associated with the primary mode interaction point, i.e., the point where the two resonance tongues intersect $[2,3]$; it takes place between the modes $1 \alpha$ and $2 \beta$ via the MP-motion, or in group theoretical language, between $Z_{2}(R T) \times Z_{2}(E) \times Z_{2}(t)$ and $Z_{2}(R T) \times Z_{2}(E T) \times Z_{2}(T t)$ via their common subgroup $Z_{2}(R T) \times R_{2}(E t)$. The second one is associated with the secondary mode interaction point [3] and takes place between the MP- and A-motions via their common daughter ML, or in group language, between $Z_{2}(R T) \times Z_{2}(E t)$ and $Z_{2}(R T) \times Z_{2}(t)$ via the subgroup $Z_{2}(R T)$. In the dissipative case only the first of these two interactions survives. Here, in the absence of time-reversal symmetry, the groups of the interacting modes are $Z_{2}(R T) \times Z_{2}(E)$ and $Z_{2}(R T) \times Z_{2}(E T)$, and their common daughter has symmetry group $Z_{2}(R T)$. The second interaction disappears, since the motions A and MP now fall within the same symmetry class $Z_{2}(R T)$; the only remnant of the former interaction is the saddle-node bifurcation separating these two motions (see also [3]).

The dissipative lattice is a collapsed version of the Hamiltonian one. Motions that are neatly separated (on different vertices) in the Hamiltonian lattice are clustered together (on one vertex) in the dissipative case. Indeed, the saddle-node connections between 
these clustered motions in the dissipative system would be rather puzzling if one were not familiar with the Hamiltonian case. Thus, as remarked before in Ref. [1], the Hamiltonian system provides the backbone of the dissipative system. The general lesson here is that, even though the dissipative system is more directly linked with experiment, knowledge of the Hamiltonian system is indispensable for a proper understanding of the dynamics.

Another thing dictated by the symmetry of the motions, as shown in Section 4, is whether or not they can undergo period doubling- and Hopf bifurcations. The presence of a spatio-temporal symmetry with temporal part $\boldsymbol{T}$ (in our system: $\boldsymbol{R T}, \boldsymbol{R E}$ or $\boldsymbol{E T}$ ) prohibits the motion from undergoing a period doubling bifurcation. Likewise, a pure spatial symmetry forms an obstacle for a Hopf bifurcation, and in the two-pendulum system already one such symmetry $(\boldsymbol{R}, \boldsymbol{E}$ or $\boldsymbol{R E})$ is enough to make a Hopf bifurcation impossible. Applying these (general) results to the system under consideration one finds that escape from the isotropy lattice, i.e., a transition to oscillations which do not have periodicity $2 T$, is not possible before the third level (in agreement with the observations in the three previous papers [1-3]). The lattice therefore captures the better part of the bifurcation sequences observed in the system.

In general, for systems with many degrees of freedom, period doubling bifurcations may be anticipated to be rarer than Hopf bifurcations. To prohibit a period doubling one single spatio-temporal symmetry (of the type described above) is enough. A Hopf bifurcation, on the other hand, is not completely obstructed unless the motion is confined to a (two-dimensional) plane in stroboscopic phase space; this generally takes more than one spatial symmetry. In this context it is also worth noting that, in systems governed by many-dimensional equations of motion, the route to chaos via successive period doubling bifurcations without the assisting intervention of a Hopf bifurcation is (if it exists at all) normally restricted to a very narrow path through parameter space. We have already come across this fact for the case of the $1 \alpha$-motion in Refs. [1,2].

Finally, the symmetry approach enables a meaningful comparison with other systems exhibiting mode interaction. Most of the experimental observations of mode interaction have been done in systems with many degrees of freedom and complicated equations of motion [4,5,8-14]; a comparison with these systems on the level of the detailed equations is difficult and hardly adds anything to the understanding of the phenomenon. On the level of the symmetry properties, however, such a comparison is both simpler and more rewarding. Indeed, the unifying principle of mode interaction then presents itself almost automatically: mode interaction can only occur between those modes that have a common daughter in the system's symmetry hierarchy.

Of course, we do not mean to say that the equations of motion are unimportant, only that comparisons between different systems on that level tend to get convoluted. The specific features of the interaction (such as the position of the interaction region in parameter space, the stability of the interaction mode and the bifurcations it undergoes) depend heavily on the nonlinear terms in the equations of motion. An illustration of this is provided by the compound pendulum discussed in Section $5[6,7]$, which has exactly the same isotropy lattice as our system but for which the actual appearance 
of the mode interaction is quite different. Here, as usual, the equivalence of the mode interaction only becomes transparent on the more abstract level of symmetry groups.

\section{Acknowledgements}

We would like to thank M.T. Westra for a fruitful discussion concerning the role of symmetry in the obstruction of period doubling bifurcations. We further thank J.C. Ross for his continuing interest and many stimulating conversations.

\section{Appendix. The structure of the Hamiltonian isotropy lattice}

An elegant way of representing the 41 groups of the Hamiltonian isotropy lattice (see Section 2 in the main text) is by means of a three-dimensional graph. The vertices of this graph stand for the groups and the connecting lines represent the allowed symmetry breaking bifurcations. In this appendix we shall construct the graph step by step.

The first step is from the trivial motion, at level I, to the pure modes of level II. The natural way to position the four (equivalent) level II vertices is in a tetrahedral structure surrounding the level I vertex. This yields Fig. 12a, in which the level I vertex has been depicted as a sphere and the level II vertices as cubes. The groups represented by the vertices have been indicated in shorthand notation; $\boldsymbol{R T} \times \boldsymbol{E} \times \boldsymbol{t}$, for instance, stands for the group $Z_{2}(R T) \times Z_{2}(E) \times Z_{2}(t)$, with elements $R T, E, t, R T E, R T t, E t, R T E t$ and 1. Throughout the isotropy lattice, vertices corresponding to motions that have actually been observed (and discussed in Refs. [1-3]) will be labeled with the motion's name. In Fig. $12 \mathrm{a}$ this means that the level I vertex carries the name of the 0 -motion, and the level II vertices those of the normal motions $1 \alpha, 2 \alpha, 1 \beta$ and $2 \beta$.

Fig. $12 \mathrm{~b}$ shows the next step, that is, the transition from level II to level III. The vertices of level II are (again) depicted as cubes and those of level III as gray spheres. Each of the spheres is accompanied by the three group elements that, together with the identity $\mathbf{1}$, constitute the symmetry group it represents.

The level II vertices each have seven daughters in level III, which can be divided into three categories. The first category consists of four vertices, singled out by the fact that none of their group elements contains the operation $\boldsymbol{R}$. As an example from this category we see the mixed motion type D (with elements ET, $\boldsymbol{t}, \boldsymbol{E T t}$ and 1) right above its mother $2 \alpha$. Together the vertices of this category form a new, large tetraeder.

The second category consists of 12 vertices, positioned in four crown-like structures placed on top of the level II vertices; the vertices of the first category form the centers of these crowns. The second category is characterized by the fact that $\boldsymbol{R}$ is present (in combination with other symmetry operations) but never as the element $\boldsymbol{R T}$. An example is the nonsymmetrical $2 \alpha$-motion (with elements $\boldsymbol{R E}, \boldsymbol{t}, \boldsymbol{R E t}$ and 1 ) in the upper left part of the picture.

The third category consists of the remaining six vertices. In this category $\boldsymbol{R}$ occurs in combination with all other symmetries (the identity $\mathbf{1}$ excepted, i.e., $\boldsymbol{R}$ never occurs 

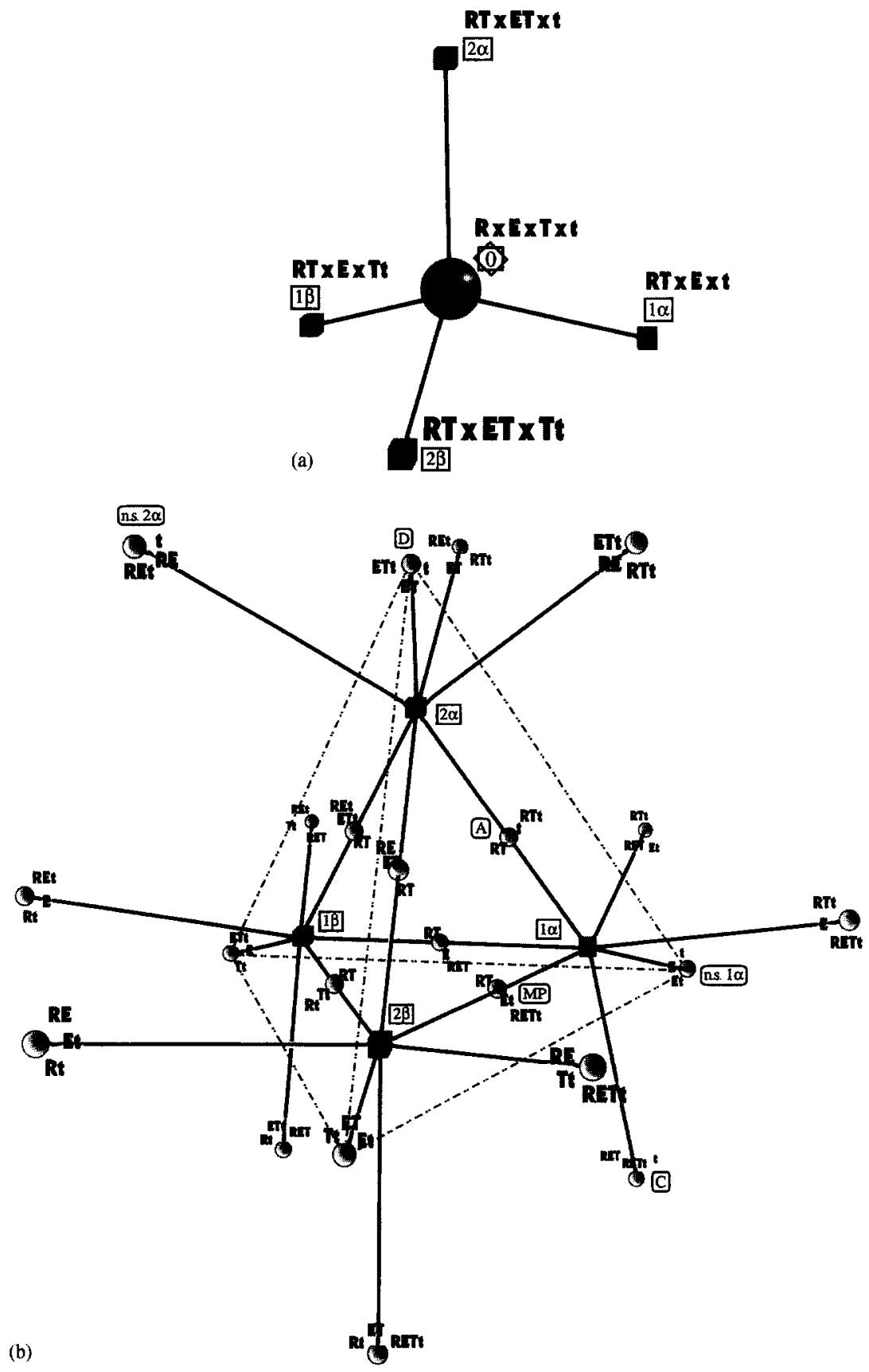

Fig. 12. The Hamiltonian isotropy lattice. 


$$
\checkmark
$$


alone) and moreover, every vertex in this category contains the element $\boldsymbol{R T}$. These vertices are of special importance since they have two mothers (in level II) and hence represent possible interaction modes between them. In the figure these interaction-vertices are positioned halfway between their two mothers. For example, the vertex labeled as MP is a daughter of both the $1 \alpha$ - and the $2 \beta$-vertex, and indeed we know from Ref. [3] that the MP-motion establishes the interaction between the $1 \alpha$ - and the $2 \beta$-motion. It should be noted, however, that not all mode interactions suggested by the isotropy lattice are realized in practice. More specifically, of the six interaction-vertices in Fig. $12 b, M P$ is the only one which has actually been observed to act as an interaction mode between its two mothers (the $1 \alpha$ - and $2 \beta$-motion) in our two-pendulum system. As for the A-motion its connection with the $1 \alpha$-motion has been observed (and indeed plays an important role in our system [1-3]) but not its connection with the $2 \alpha$-motion, and so it has never been seen to act as an interaction mode between the two.

For each vertex in level III there are three different ways of writing the group structure (since every group contains three nontrivial elements). In the left column of Table 1 in the main text we have chosen a notation which economizes the number of generators per category. Consider the seven daughters of a level II vertex. The daughter that falls into the first category (the center of the crown) is, of course, determined by two generators. The three daughters that fall in the second category (the crown itself) can be specified cyclically by three generators. Finally, to describe the three daughters that fall in the third category (the interaction modes) four generators are needed. This notation is the most elegant one from a theoretical point of view.

An alternative notation is presented in the middle column of Table 1 . This notation anticipates Section 3, where the (Hamiltonian) system is perturbed by dissipation, which causes the time-reversal symmetry $\boldsymbol{t}$ to disappear. In this notation the operator $\boldsymbol{t}$, if it appears at all, is put in one generator only; thus, the group structure of the level III vertices in the dissipative system can immediately be obtained from the middle column of Table 1 by simply omitting the generator containing $t$. This is also true (without the need of an alternative notation) for the vertices of the other levels.

We then go on to Fig. 12c, displaying the transition from level III to level IV. Each of the level III vertices (the gray spheres) has three daughters in the next level, numbering up to a total of 13 , as already mentioned in the main text; the latter have been indicated by dark spheres. They lie on three orthogonal axes through the centre of the lattice, in such a way that the tetrahedral symmetry of the structure is preserved. The symmetry groups represented by the dark spheres consist of only two elements, namely the identity $\mathbf{1}$ and the element indicated in the figure. It may be noted that the six outer vertices and the vertex in the center all contain $\boldsymbol{R}$, while the six vertices in between do not. Furthermore, every vertex in level IV is intimately related to its mirror-vertex (with respect to the center of the tetrahedral structure), the one being converted into the other by the operation $\boldsymbol{T}$. These properties reflect the special status (in our lattice) of the $\boldsymbol{R}$ and $\boldsymbol{T}$ operations; we shall come back to this briefly at the end of the appendix. 
As always, the solid lines between the vertices of level III and IV represent the mother-daughter connection. ${ }^{7}$ It is easily verified from this picture that each daughter in level IV has five mothers in level III and the central daughter, RT, even has six mothers; as a consequence we have a plethora of possible mode interactions. Again, this does not mean that all the interactions suggested by the lattice are actually observed in practice. In fact, only the ML-motion (corresponding to the central vertex $\boldsymbol{R T}$ ) has been observed to act as an interaction mode between two of its mothers, namely between the A- and the MP-motion [3].

The wireframe of all the mother-daughter connections in Fig. 12 is quite complicated, and it is not easy to discern the geometric pattern. To guide the eye, therefore, a 'solid' version is presented in Fig. 12d. This figure consists of a tetraeder cut through by three orthogonal planes; the resulting structure has perfect tetrahedral symmetry (just as Fig. $12 \mathrm{a}$ and Fig. 12b). The symmetry is even more apparent when we take a view from right above one of the tops of the tetraeder, as in Fig. 13a, or from a point just above an intersection point of two orthogonal planes, as in Fig. 13b. These viewpoints have been indicated in Fig. 12d by the pointing hands. Fig. 13a and Fig. 13b nicely illustrate the symmetry of the structure but they also have a weak side: some vertices lie exactly behind others and are therefore obscured. This is the reason why in the main pictures (Fig. 12a-Fig. 12c) we have chosen another, less biased viewpoint.

For the sake of completeness it should be noted that all 13 groups of level IV have one and the same daughter, namely the identity 1 , which by itself constitutes level V. The corresponding picture would consist of the 13 dark spheres, connected by 13 solid lines to a single vertex in the middle. The motions corresponding to this identity-vertex are still $2 T$-periodic oscillations, but do not possess any of the symmetries considered.

We now have an overview of the isotropy lattice, and we have seen time and again that its structure is dictated to a large extent by the operations $\boldsymbol{R}$ and $\boldsymbol{T}$. They never appear as single elements (whereas $\boldsymbol{E}$ and $\boldsymbol{t} d o$ ) and, moreover, their combination $\boldsymbol{R T}$ appears right in the center of the tetrahedral structure. This special status is the group-technical expression of the fact that we have chosen to consider (right from the start, in Fig. 12a) only those groups that represent oscillations bifurcating from the 0 -motion at the first-order resonance tongues. In terms of group theory this means that we have taken $Z_{2}(R) \times Z_{2}(E) \times Z_{2}(T) \times Z_{2}(t)$ to represent the 0 -motion and subsequently considered only those four maximal subgroups that contained neither $\boldsymbol{R}$ nor $\boldsymbol{T}$ as single elements (but, as a consequence, always the combination $\boldsymbol{R} \boldsymbol{T}$ ). At the next stage, in Fig. 12b, it means that only those level III vertices that contain the element $\boldsymbol{R T}$ serve as interactions between level II vertices. One step further in the lattice, it explains why level IV (depicted in Fig. 12c) consists of 13 vertices: this number is equal to 16 (the number of elements of the original $Z_{2} \times Z_{2} \times Z_{2} \times Z_{2}$ group) minus 2 (the two 'absent' vertices $\boldsymbol{R}$ and $\boldsymbol{T}$ ) minus 1 (the identity 1 ). Finally,

\footnotetext{
${ }^{7}$ There is one intricacy here: the connection from an interaction vertex of level III (e.g., the one that is positioned between $\boldsymbol{R T}$ and $\boldsymbol{t}$, labeled 'A' in Fig. 12b) towards the outer level IV vertex on its axis (RTt) seems to establish a mother-daughter connection between the two level IV vertices on the axis (RTt and $t$ ). This is, of course, an artifact of the drawing.
} 


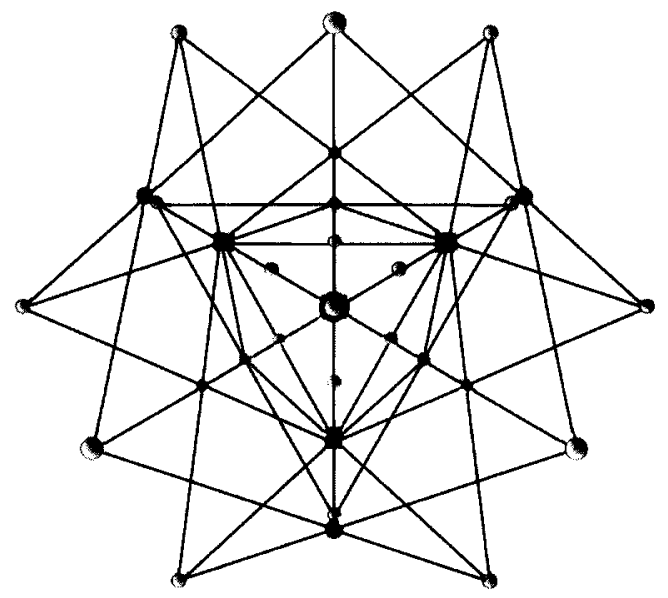

(a)

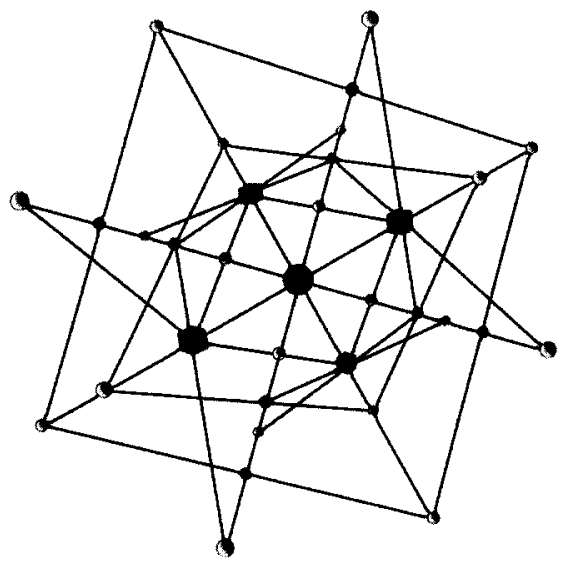

(b)

Fig. 13. The Hamiltonian isotropy lattice viewed from, as indicated in Fig. 12d, (a) the top of the tetraeder. (b) the intersection of two orthogonal planes.

it is the reason why $\boldsymbol{R T}$ and 1, i.e., the elements common to all four pure modes in level I, eventually turn up (at the levels IV and V) in the very centre of the symmetry structure.

\section{References}

[1] E.J. Banning, J.P. van der Weele, Mode competition in a system of two parametrically driven pendulums, the Hamiltonian case, Physica A 220 (1995) 485-533.

[2] E.J. Banning, J.P. van der Weele, J.C. Ross, M.M. Kettenis, E. de Kleine, Mode competition in a system of two parametrically driven pendulums; the dissipative case, Physica A 245 (1997) 11-48. 
[3] E.J. Banning, J.P. van der Weele, J.C. Ross, M.M. Kettenis, Mode competition in a system of two parametrically driven pendulums with nonlinear coupling, Physica A 245 (1997) 49-98.

[4] S. Ciliberto, J.P. Gollub, Pattern competition leads to chaos, Phys. Rev. Lett. 52 (1984) 922-925.

[5] S. Ciliberto, J.P. Gollub, Chaotic mode competition in parametrically forced surface waves, J. Fluid Mech. 158 (1985) 381-398.

[6] A.C. Skeldon, T. Mullin, Mode interaction in a double pendulum, Phys. Lett. A 166 (1992) 224-229.

[7] A.C. Skeldon, Dynamics of a parametrically excited double pendulum, Physica D 75 (1994) 541-558.

[8] H. Ayanle, A. Bernoff, S. Lichter, Spanwise modal competition in cross-waves, Physica D 43 (1990) $87-104$.

[9] L. Shemer, S. Lichter, The mode number dependence of neutral stability of cross-waves, Experiments in Fluids 9 (1990) 148-152.

[10] W.B. Underhill, S. Lichter, A.J. Bernoff, Modulated, frequency-locked, and chaotic cross-waves, J. Fluid Mech. 225 (1991) 371-394.

[11] J.R. Buchler, T. Serre, Z. Kollath, J. Mattei, A chaotic pulsating star: the case of R Scuti, Phys. Rev. Lett. 73 (1995) 842-845.

[12] H. Levine, W.J. Rappel, H. Riecke, Resonant interactions and traveling-solidification cells, Phys. Rev. A 43 (1991) 1122-1125.

[13] R. Grauer, Codimension two interactions of tearing modes, in: St. Pnevmatikos, T. Bountis, Sp. Pnevmatikos (Eds.), Proc. Internat. Conf. on "Singular Behavior and Nonlinear Dynamics, vol. I., Samos (Greece), 1988. World Scientific, Singapore, 1989, pp. 267-276.

[14] S.M. Cox, Mode interactions in Rayleigh Bénard convection, Physica D 95 (1) (1996) 50-61.

[15] M. Golubitsky, I. Stewart, D.G. Schaeffer, Singularities and Groups in Bifurcation Theory, Vol. II, Applied Mathematical Sciences, vol. 69 Springer, New York, 1988.

[16] J.P. van der Weele, E.J. Banning, Mode interaction in a cup of coffee and other nonlinear oscillators, Open Systems Inform. Dyn., vol. 5, Kluwer, 1998.

[17] J.E. Howard, R.S. MacKay, Linear stability of symplectic maps, J. Math. Phys. 28 (1987) 1036-1051.

[18] E. Ott, Chaos in Dynamical Systems, Cambridge University Press, Cambridge, 1993.

[19] Y.A. Kuznetsov, Elements of Applied Bifurcation Theory, Applied Mathematical Sciences, Vol. 112, Springer, Berlin, 1995.

[20] J. Argyris, G. Faust, M. Haase, An Exploration of Chaos, Texts on Computational Mechanics, Vol. VII, Elsevier, Amsterdam, 1994.

[21] E. Meron, I. Procaccia, Low-dimensional chaos in surface waves: theoretical analysis of an experiment, Phys. Rev. A 34 (1986) 3221-3237.

[22] E. Meron, I. Procaccia, Theory of chaos in surface waves: the reduction of hydrodynamics to few-dimensional dynamics, Phys. Rev. Lett. 56 (1986) 1323-1326.

[23] E. Meron, Parametric excitation of multimode dissipative systems, Phys. Rev. A 35 (1987) 4892-4895.

[24] M. Umeki, T. Kambe, Nonlinear dynamics and chaos in parametrically excited surface waves. J. Phys. Soc. Japan 58 (1989) 140-154.

[25] H.W. Müller, H. Wittmer, C. Wagner, J. Albers, K. Knorr, Analytic stability theory for Faraday waves and the observation of the harmonic surface response, Phys. Rev. Lett. 78 (1997) 2357-2360, and references therein.

[26] J.D. Crawford, E. Knobloch, H. Riecke, Period-doubling mode interactions with circular symmetry, Physica D 44 (1990) 340-396.

[27] Th. Zeegers, On the existence of infinite period-doubling sequences in a class of $4 \mathrm{D}$ semi-symplectic mappings, J. Phys. A 24 (1991) 2287-2314. 